\title{
Network Pharmacology to Uncover the Biological Basis of Spleen Qi Deficiency Syndrome and Herbal Treatment
}

\author{
Xin Wang, ${ }^{1}$ Min Wu, ${ }^{1}$ Xinxing Lai $\left(\mathbb{D},{ }^{1}\right.$ Jiahui Zheng, ${ }^{1}$ Minghua $\mathrm{Hu}^{2}{ }^{2}$ Yan $\mathrm{Li}^{3}$ and Shao $\mathrm{Li}^{\mathrm{D}}{ }^{1}$ \\ ${ }^{1}$ MOE Key Laboratory of Bioinformatics, Bioinformatics Division and Center for TCM-X, BNRIST / Department of Automation, \\ Tsinghua University, Beijing 100084, China \\ ${ }^{2}$ Infinitus (China) Company Ltd., LKK Health Products Group, 510623, China \\ ${ }^{3}$ State Key Laboratory of Bioactive Substances and Functions of Nature Medicines, Institute of Materia Medica, Chinese Academy of \\ Medical Sciences \& Peking Union Medical College, 100050 Beijing, China
}

Correspondence should be addressed to Xinxing Lai; laixinxing@mail.tsinghua.edu.cn and Shao Li; shaoli@tsinghua.edu.cn

Received 16 April 2020; Accepted 28 May 2020; Published 27 August 2020

Guest Editor: Yue Liu

Copyright (c) 2020 Xin Wang et al. This is an open access article distributed under the Creative Commons Attribution License, which permits unrestricted use, distribution, and reproduction in any medium, provided the original work is properly cited.

Spleen qi deficiency (SQD) syndrome is one of the basic traditional Chinese medicine (TCM) syndromes related to various diseases including chronic inflammation and hypertension and guides the use of many herbal formulae. However, the biological basis of SQD syndrome has not been clearly elucidated due to the lack of appropriate methodologies. Here, we propose a network pharmacology strategy integrating computational, clinical, and experimental investigation to study the biological basis of SQD syndrome. From computational aspects, we used a powerful disease gene prediction algorithm to predict the SQD syndrome biomolecular network which is significantly enriched in biological functions including immune regulation, oxidative stress, and lipid metabolism. From clinical aspects, SQD syndrome is involved in both the local and holistic disorders, that is, the digestive diseases and the whole body's dysfunctions. We, respectively, investigate SQD syndrome-related digestive diseases including chronic gastritis and irritable bowel syndrome and the whole body's dysfunctions such as chronic fatigue syndrome and hypertension. We found innate immune and oxidative stress modules of SQD syndrome biomolecular network dysfunction in chronic gastritis patients and irritable bowel syndrome patients. Lymphocyte modules were downregulated in chronic fatigue syndrome patients and hypertension patients. From experimental aspects, network pharmacology analysis suggested that targets of Radix Astragali and other four herbs commonly used for SQD syndrome are significantly enriched in the SQD syndrome biomolecular network. Experiments further validated that Radix Astragali ingredients promoted immune modules such as macrophage proliferation and lymphocyte proliferation. These findings indicate that the biological basis of SQD syndrome is closely related to insufficient immune response including decreased macrophage activity and reduced lymphocyte proliferation. This study not only demonstrates the potential biological basis of SQD syndrome but also provides a novel strategy for exploring relevant molecular mechanisms of disease-syndrome-herb from the network pharmacology perspective.

\section{Introduction}

Understanding the biological basis of syndromes ("ZHENG” in Mandarin Chinese) is an essential component of traditional Chinese medicine (TCM) modernization. Spleen qi deficiency (SQD) syndrome is one of the most common TCM syndromes and is characterized by fatigue, abdominal distension, boredom, and other phenotypes [1,2]. However, the current research of SQD syndrome is somewhat limited [3] and may not be suitable for elucidating the biological basis of SQD syndrome at a systematic level. Some studies have explored the biological basis of some typical syndromes in TCM such as Cold syndrome and Hot syndrome. Based on network pharmacology analysis, Cold syndrome and Hot syndrome are closely associated with the metabolismimmune imbalance [4]. The biological networks underlying Cold syndrome and Hot syndrome have been applied to clinical investigation by integrating clinical transcriptional profiles. The network modules underlying Cold syndrome indicate that energy metabolism decreased in Cold syndrome 
patients [5]. In addition, the Hot syndrome biomolecular network suggests that inflammatory response increased in chronic gastritis with Hot syndrome [6]. These findings provide an important foundation at the molecular and systematic level for studying other typical syndromes such as SQD syndrome.

SQD syndrome-related research mainly contains clinical observations and experiments. In clinical terms, according to the holistic perspective of TCM, SQD syndrome not only refers to local digestive diseases such as chronic gastritis and irritable bowel syndrome but also contains the whole body's dysfunctions such as chronic fatigue syndrome and hypertension [7-9]. In experimental terms, animal models have been constructed by reserpine injection or irregular food administration [10]. Understanding the biological basis of SQD syndrome may guide the evidence-based clinical use of herbal formulae. Herbal formulae such as Si-Jun-Zi decoctions in China's National Basic Medical Insurance Drug Catalogue are widely used clinically to treat chronic gastritis and other SQD syndrome-related diseases [11, 12]. Most of the studies on these herbal formulae have focused on immunity, antioxidation, and metabolism [13-15]. Revealing the biological basis of SQD syndrome from a systematic perspective will be of great help to precision medicine for TCM syndrome-related diseases.

Given the advent of the artificial intelligence and big data era, the rapid development of information science and omics technology has provided a solid foundation for moving beyond the limitations of current medical research methods and establishing new network-based approaches $[4,16]$. In recent years, the "network target, multicomponent therapeutics" approach was proposed for investigating complex diseases and herbal formulae [17]. A network target is a core principle in the network pharmacology. Different from the "one target, one drug" paradigm, the network target refers to a novel concept that treats the biological network underlying diseases and TCM syndromes as a therapeutic target in order to decipher systematic mechanisms of action for multitarget drugs and herbal formulae [18]. More and more evidence shows that the network target approach is suitable for elucidating the biological basis of the TCM syndrome and herbal treatment. For example, the network target approach is used to detect antirheumatic mechanisms of the TCM formula Qing-Luo-Yin for treating Hot syndrome-related rheumatoid arthritis [19] and unveil the molecular mechanisms of Ge-Gen-Qin-Lian decoction, which is an ancient and effective herbal formula for "dampness heat" syndrome type II diabetes [20].

In this article, based on network target theory, we further propose a network pharmacology approach integrating computational, clinical, and experimental investigation to elucidate biological associations between SQD syndromes, diseases, and herbal treatments (Figure 1). First, we computationally predicted the SQD syndrome-related biomolecular network. And then, we further study the biological functions of this network including immune and oxidative stress from clinical transcriptomic data of SQD syndrome-related diseases such as chronic gastritis and hypertension. Furthermore, network pharmacology analysis suggested that the targets of Radix Astragali, Rhizoma Atractylodis Macrocephalae, Radix Codonopsis Pilosulae, Radix Ginseng, and Rhizoma Dioscoreae commonly used for SQD syndrome are significantly enriched in the SQD syndrome biomolecular network. Experiments further validated that Radix Astragali ingredients promoted immune modules of the SQD syndrome biomolecular network. The network pharmacology strategy could provide a new approach to transform experience-based TCM syndrome into biological networkbased TCM precision medicine.

\section{Materials and Methods}

2.1. Methods for Prediction of the SQD Syndrome Biomolecular Network. In this work, we predicted the SQD syndromerelated biomolecules at the genome-wide level using the CIPHER algorithm with 14 clinical phenotypes of SQD syndrome (Supplementary Table S1). The top 500 biomolecules were selected to form the SQD-related biomolecule list according to the high accuracy of the algorithm. According to the predicted biomolecules, protein-protein interactions, and signaling pathways, we generated the SQD syndrome biomolecular network. CIPHER was a powerful disease gene network-based prediction algorithm [21]. In principle, this algorithm explores the modularity of human phenotypebiomolecule through network-based integration of multiple phenotype similarities among OMIM-recorded diseases and TCM syndromes and protein-protein interactions (PPIs) among candidate biomolecules. This network pharmacology algorithm has been robustly evaluated in the international journal and is used to identify the clinical biomarkers [22, 23].

2.2. Computational Validation of the SQD Syndrome Biomolecular Network. In mining for literature on SQD syndrome or compounds, we searched the PubMed database using the keywords "Spleen Qi deficiency syndrome" or the compound name in the abstract and recorded the total number of search results. We downloaded the identified abstracts and extracted the biomolecules listed in the abstracts using a text processing program. For the reliability of CIPHER prediction for SQD syndrome-related biomolecules, we randomly shuffled the protein-protein interaction (PPI) network 1000 times to calculate concordance scores to predict phenotype-gene relationships. The PPI network was generated with 137,037 interactions among 13,388 biomolecules. We selected 500 biomolecules as predicted biomolecules of SQD syndrome in the network each time. The literature mining was performed by the open-source programming language Ruby (version 2.3.0). If a biomolecule cooccurred in the abstract with the SQD syndrome or compound name, we manually verified and considered that the biomolecule is related to SQD syndrome and herbal ingredients. We calculated the recall for predictions and precision as recall $=[$ the intersection of predicted biomolecules and reported biomolecules/reported biomolecules] $\times 100 \%$ and precision $=[$ the intersection of predicted biomolecules and reported biomolecules/predicted biomolecules] $\times 100 \%$, respectively. 


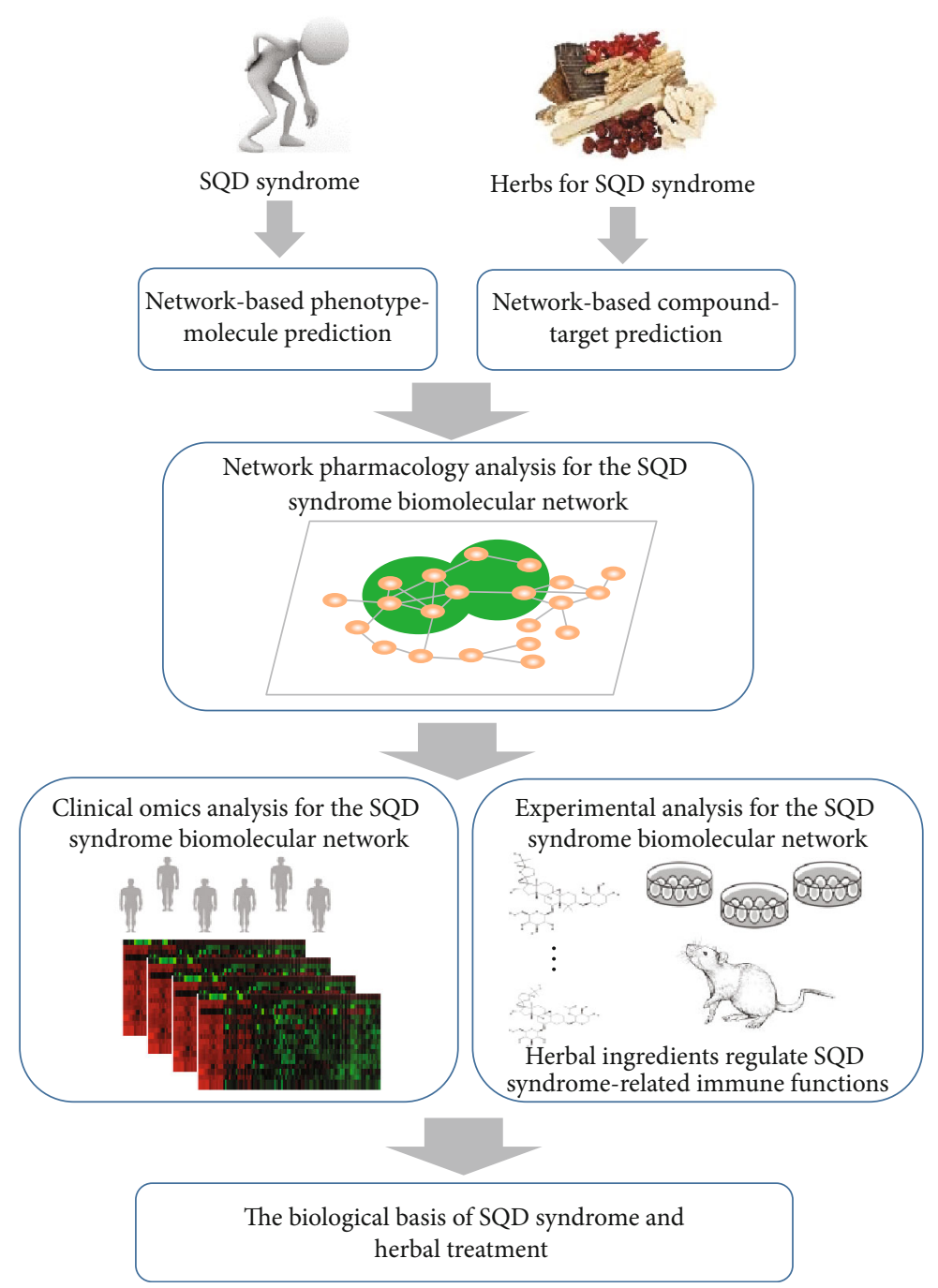

FIGURE 1: A workflow of the network pharmacology strategy for uncovering the biological basis of SQD syndrome and herbal treatment.

2.3. Biological Function Enrichment Analysis of the SQD Syndrome Biomolecular Network. We performed biological function enrichment analysis for predicted biomolecules related to SQD syndrome. The enrichment significance of the predicted biomolecules in the gene sets of GO biological processes or KEGG signaling pathways was determined using Fisher's exact test $[24,25]$. Significantly enriched biological processes and pathways $(P<0.05$, Benjamin correction) were selected for further investigation. The biological function enrichment analysis was performed using open-source programming languages $\mathrm{R}$ (version 3.6.1) and Ruby (version 2.3.0).

2.4. Clinical Transcriptomic Data Analysis of SQD SyndromeRelated Diseases. We collected gene expression profiles in gastric tissue samples from patients with Cold syndrome (diagnosed as "Spleen Stomach Deficiency Cold (SSDC) syndrome") or Hot syndrome (diagnosed as "Spleen Stomach Dampness Hot (SSDH) syndrome") [6]. These samples were assessed using the Affymetrix Human Genome U133 Plus 2.0 Array (U133Plus2.0, Affymetrix, Inc.). The probe signals were generated from the original expression data file via a standardization protocol provided by dChip software [26]. Gene expression data for chronic fatigue syndrome (CFS), irritable bowel syndrome (IBS), and hypertension patients (GSE14577, GSE14841, and GSE75360) were obtained from the GEO database [27]. The selected differentially expressed genes were connected to predicted biomolecules of SQD syndrome by a direct or indirect relationship (including proteinprotein interactions or signaling pathway) and related to the enriched biological functions of the SQD syndrome biomolecular network.

2.5. Target Prediction of Herbal Ingredients for SQD Syndrome. Firstly, we collected the ingredients of five herbs for SQD syndrome by chemical analysis experiments from literature [28-32], including ingredients from Radix Astragali, Rhizoma Atractylodis Macrocephalae, Radix Codonopsis Pilosulae, Radix Ginseng, and Rhizoma Dioscoreae. After filtering redundant information, 252 ingredients were obtained. Then, we extracted the chemical structure of each ingredient in the PubChem database for further target 
prediction by the drugCIPHER algorithm. The ingredients that meet the druglikeness standard (weighted Quantitative Estimate of Druglikeness (wQED) > 0.05) were chosen for further computational analysis. The wQED is calculated by the following formula [33]:

$$
\mathrm{wQED}=\exp \left(\frac{\sum_{i=1}^{n} \omega_{i} \ln d_{i}}{\sum_{i=1}^{n} \omega_{i}}\right),
$$

where $d$ is the individual desirability function, $\omega$ is the weight applied to each function, and $n$ is the number of descriptors.

The potential targets of the selected ingredients were predicted by drugCIPHER [34], a network pharmacology algorithm developed for the prediction of compound targets. In principle, by integrating the association of the given compound with FDA-approved drugs and the association of known targets of FDA-approved drugs in the PPI network, drugCIPHER predicts a target list of each compound. The likelihood of the compound-target interaction is defined as

$$
\Phi_{p}=\beta_{p}^{\prime}+\sum_{d j \in B(p)} \alpha_{p d j}^{\prime} \mathbf{C S}_{d j}
$$

where $d_{j}$ is the known drug $j$ binding to the given protein $p$. $\beta^{\prime} p$ and $\alpha_{p d j}^{\prime}$ are the model coefficients. $\mathbf{C S}_{d}$ is the similarity vector of the compound $d$ and the known drugs in the algorithm model. According to the concordance score of each compound-target, drugCIPHER prioritizes the proteins in the PPI network, and the candidate proteins with high concordance score $\rho_{p d}$ are hypothesized to be putative targets of the compound by the following formula:

$$
\rho_{p d}=\frac{\operatorname{cov}\left(\mathbf{C S}_{d}, \Phi_{p}\right)}{\sigma\left(\mathbf{C S}_{d}\right) \sigma\left(\Phi_{p}\right)} .
$$

Compounds and proteins with high concordance scores are more likely to exhibit drug-target interactions. The top 100 predicted targets of each compound are selected as potential targets according to the high accuracy of the drugCIPHER algorithm.

Some targets may appear in the target lists of many ingredients in an herb for SQD syndrome. To assess the probability of target proteins being related to the herb for SQD syndrome pharmacological effects, we compared the number of occurrences of each target in the target list of all ingredients. A statistical model was established to compare the number of occurrences of a target protein for each herb with that in a random background; this analysis yielded the significantly frequently occurring targets $(P<0.05)$ as the targets of each herb [35]. The random process is represented by the Poisson binomial statistical model:

$$
\operatorname{Pr}(K=k)=\sum_{A \in F_{k}} \prod_{i \in A} p_{i} \prod_{j \in A^{c}}\left(1-p_{j}\right),
$$

where $\operatorname{Pr}(K=k)$ is the probability that a protein occurs in the predicted target list of $k$ ingredients, $F_{k}$ is all subsets of $k$ ingredients, $A$ is one subset of $k$ ingredients, and $A^{c}$ is the complement of subset $A$. In addition, $p_{i}$ and $p_{j}$ are the probabilities of a protein being contained in the predicted target list of an ingredient and are calculated as [the number of potential targets/the number of all candidate targets] in the random case. $i$ and $j$ are used to distinguish different compounds. The $P$ value $\operatorname{Pr}(K>k)$ measures the probability of a target occurring in more than $k$ ingredients' target lists by 1000 random cases. This adjusted $P$ value indicates the significance of targets of herbs for SQD syndrome $(P$ value $<$ 0.05 is significant). The target prediction of herbs for SQD syndrome was performed by the opensource programming language $\mathrm{R}$ (version 3.6.1).

\subsection{Experimental Materials and Methods for the SQD Syndrome Biomolecular Network}

2.6.1. Reagents. Astragaloside I and astragaloside II were purchased from Nanjing Jingzhu Bio-Technology Co., Ltd. Astragaloside IV (98\% purity) was purchased from Chengdu Herbpurify Co., Ltd., and astragalus polysaccharides (70\% purity) were purchased from Shanghai Macklin Co., Ltd. The compounds were dissolved in dimethyl sulfoxide (DMSO) (final concentration $<0.1 \%$ ), which was used as the solvent control for experiments. For experiments, compounds were dissolved in an alkaline solution. Penicillin sodium salt and streptomycin sulfate were purchased from North China Pharmaceutical Co., Ltd. DMEM and trypsin were obtained from Gibco. RPMI-1640 medium was obtained from Beijing Keyin Watson Scientific Development Co., Ltd., and foetal bovine serum was obtained from Beijing Yuanshang Shengma Biotechnology Institute. DMSO was produced by Beijing Chemical Plant. Tetrazolium bromide (MTT) was provided by Beijing Soleble Technology Co., Ltd. ConA and LPS were purchased from Sigma-Aldrich.

2.6.2. Cell Culture. The mouse macrophage cell line RAW264.7 was a gift from Professor Wang Wenjie (Institute of Materia Medica, Chinese Academy of Medical Sciences). The cells were cultured in DMEM supplemented with $10 \%$ foetal bovine serum and $100 \mathrm{U} / \mathrm{ml}$ penicillin in an incubator containing $5 \% \mathrm{CO}_{2}$ at $37^{\circ} \mathrm{C}$. All cells were digested with $0.25 \%$ trypsin-EDTA and passaged twice a week.

2.6.3. Macrophage Proliferation of Herbs for SQD Syndrome. Raw264.7 cells were seeded in a 96-well plate at a concentration of $5 \times 10^{4}$ cells $/ \mathrm{ml}$. After a $24 \mathrm{~h}$ incubation, the cells were treated with $5 \mu \mathrm{M}$ or $50 \mu \mathrm{M}$ astragaloside I (wQED $=0.131$ ), astragaloside II $\quad(\mathrm{wQED}=0.131)$, astragaloside IV $(w Q E D=0.131)$ and astragalus polysaccharide $(w Q E D=0.571)$. Then, the cells were placed in the incubator for $96 \mathrm{~h}$. Four hours before the end of the incubation, $50 \mu \mathrm{l}$ of MTT stock solution ( $2 \mathrm{mg} / \mathrm{ml}$; Soleble Technology, China) was added to each well. After incubation, the cells were pelleted by centrifugation $(2000 \mathrm{rpm}, 10 \mathrm{~min})$. Then, we gently decanted the cell culture medium and dried the samples with a tissue. Then, $150 \mu \mathrm{l}$ of DMSO was added to each well. After completely mixing the cells and DMSO by oscillation, the 
optical density (OD) at $570 \mathrm{~nm}$ was measured using an ELISA reader (Bio-Rad, USA).

2.6.4. Spleen Lymphocyte Proliferation of Herbs for SQD Syndrome. Herbs for SQD syndrome, that is, herbs for reinforcing spleen qi such as Radix Astragali, were often used to treat colon cancer [36]. Colon cancer has the clinical phenotypes of SQD syndrome. In order to understand the immune regulation of herbs for SQD syndrome, we conducted the spleen lymphocyte proliferation experiment in the SQD syndrome-related colon cancer model. All animal protocols conformed to the Guidelines for the Care and Use of Laboratory Animals and were approved by the Animal Care and Use Committee of the Chinese Academy of Medical Sciences and Peking Union Medical College, and Balb/c mice were purchased from Beijing Weitong Lihua Experimental Animal Technology Co., Ltd. (No. SCXK2012-0001). Balb/c female mice were housed in a controlled environment at $18 \sim 25^{\circ} \mathrm{C}$ with $50 \%$ to $70 \%$ relative humidity. C26 cells were preserved by the Department of Pharmacology, Institute of Materia Medica, Chinese Academy of Medical Sciences and Peking Union Medical Colleges. Actively growing tumour tissues were dissected, cut, and ground to generate a tumour cell suspension in sterile physiological saline $\left(5 \times 10^{7}\right.$ cells $\left./ \mathrm{ml}\right)$. Each mouse was inoculated on the back with $0.2 \mathrm{ml}$ of the cell suspension. The day after inoculation, Balb/c mice were randomly divided into two groups and administered drugs once a day for 12 days. To illustrate the herbal treatment for regulating the lymphocyte function module of the SQD syndrome biomolecular network, one group received saline as a negative control, and the other group was treated with $50 \mathrm{mg} / \mathrm{kg}$ astragaloside IV according to the results of preliminary experiments on mice.

After treatment with astragaloside IV, the mice were sacrificed by cervical dislocation, and the spleens were removed under aseptic conditions and ground in a mortar using a $1 \mathrm{ml}$ sterile syringe. The cell suspension was then transferred to a plastic centrifuge tube and pelleted. The cells were diluted to $1 \times 10^{7} \mathrm{cells} / \mathrm{ml}$, and $100 \mu \mathrm{l} /$ well cell suspension was seeded in a 96-well cell culture plate (with $3.0 \mu \mathrm{g} / \mathrm{ml}$ ConA or $5.0 \mu \mathrm{g} / \mathrm{ml}$ LPS). The cell culture plate was placed in the incubator at $37^{\circ} \mathrm{C}$ with $5 \% \mathrm{CO}_{2}$ for $48 \mathrm{~h}$. Four hours before the end of the incubation, $50 \mu \mathrm{l}$ of MTT solution ( $2 \mathrm{mg} / \mathrm{ml}$; Soleble Technology, China) was added to each well. Then, the plates were centrifuged at $2000 \mathrm{rpm}$ for 10 min to pellet the cells, and the remaining MTT solution was removed with a tissue. A total of $150 \mu \mathrm{l}$ of DMSO was added to each well. Following complete mixing, the OD at $570 \mathrm{~nm}$ was measured using an ELISA reader (Bio-Rad, USA).

\subsection{Statistical Analysis of the SQD Syndrome Biomolecular} Network. The statistical significance was performed using Student's $t$-test via GraphPad Prism 5.0 in clinical transcriptional profiles and experiments. Data are expressed as the mean \pm standard deviation in experiments. Enrichment analysis was conducted by Fisher's exact test via programming
TABLE 1: Several enriched biological processes and pathways of the SQD syndrome biomolecular network.

\begin{tabular}{|c|c|c|}
\hline Class & Biological process and pathway & $P$ value \\
\hline \multirow{7}{*}{ Immune system } & $\mathrm{T}$ cell receptor signaling pathway & $4.2 E-15$ \\
\hline & $\begin{array}{l}\text { Helper T cell proliferation } \\
\text { and differentiation }\end{array}$ & $3.2 E-15$ \\
\hline & $\begin{array}{l}\text { Macrophage proliferation } \\
\text { and differentiation }\end{array}$ & $5.6 E-11$ \\
\hline & $\begin{array}{l}\text { Antigen processing } \\
\text { and presentation }\end{array}$ & $3.2 E-4$ \\
\hline & $\begin{array}{l}\text { Leukocyte transendothelial } \\
\text { migration }\end{array}$ & $1.3 E-4$ \\
\hline & $\mathrm{B}$ cell receptor signaling pathway & $6.3 E-4$ \\
\hline & $\begin{array}{l}\text { Natural killer cell-mediated } \\
\text { bioactivity }\end{array}$ & 0.0054 \\
\hline \multirow{3}{*}{$\begin{array}{l}\text { Endocrine and } \\
\text { metabolism } \\
\text { system }\end{array}$} & Estrogen signaling pathway & $1.2 E-16$ \\
\hline & Response to oxidative stress & $7.2 E-7$ \\
\hline & $\begin{array}{l}\text { Regulation of the lipid } \\
\text { metabolic process }\end{array}$ & $5.3 E-14$ \\
\hline \multirow{2}{*}{ Nervous system } & Neurotrophin signaling pathway & $7.4 E-12$ \\
\hline & Dopaminergic synapse & $8.7 E-5$ \\
\hline \multirow[b]{2}{*}{ Digestive system } & Digestion & 0.0058 \\
\hline & $\begin{array}{l}\text { Regulation of the digestive } \\
\text { system process }\end{array}$ & 0.0084 \\
\hline
\end{tabular}

languages $\mathrm{R}$ (version 3.6.1) and Ruby (version 2.3.0). $P$ values less than or equal to 0.05 were considered significant.

\section{Results}

3.1. Computational Analysis of the SQD Syndrome Biomolecular Network. We predicted the SQD syndrome biomolecular network at the genome-wide level using the CIPHER method with 14 clinical phenotypes of SQD syndrome (Supplementary Table S1). The top 500 biomolecules were predicted to form the SQD-related biomolecular network according to the high accuracy of the CIPHER algorithm [21]. As listed in Table 1, these biomolecules are significantly enriched in biological pathways and biological processes including immunity, metabolism, endocrine biological functions such as the $\mathrm{T}$ cell receptor signaling pathway, response to oxidative stress, and regulation of the lipid metabolic process. The results indicated that the biological basis of SQD syndrome is closely related to the above biological processes, as shown in Figure 2(a).

The enrichment results of the SQD syndrome biomolecular network were further validated by literature mining. We searched for the relationship between enriched biological functions and SQD syndrome in the literature. Patients with SQD syndrome may exhibit immune disorders. We used the immune modules of the SQD syndrome-related network as a starting point to systematically understand the immune-related biological basis of 


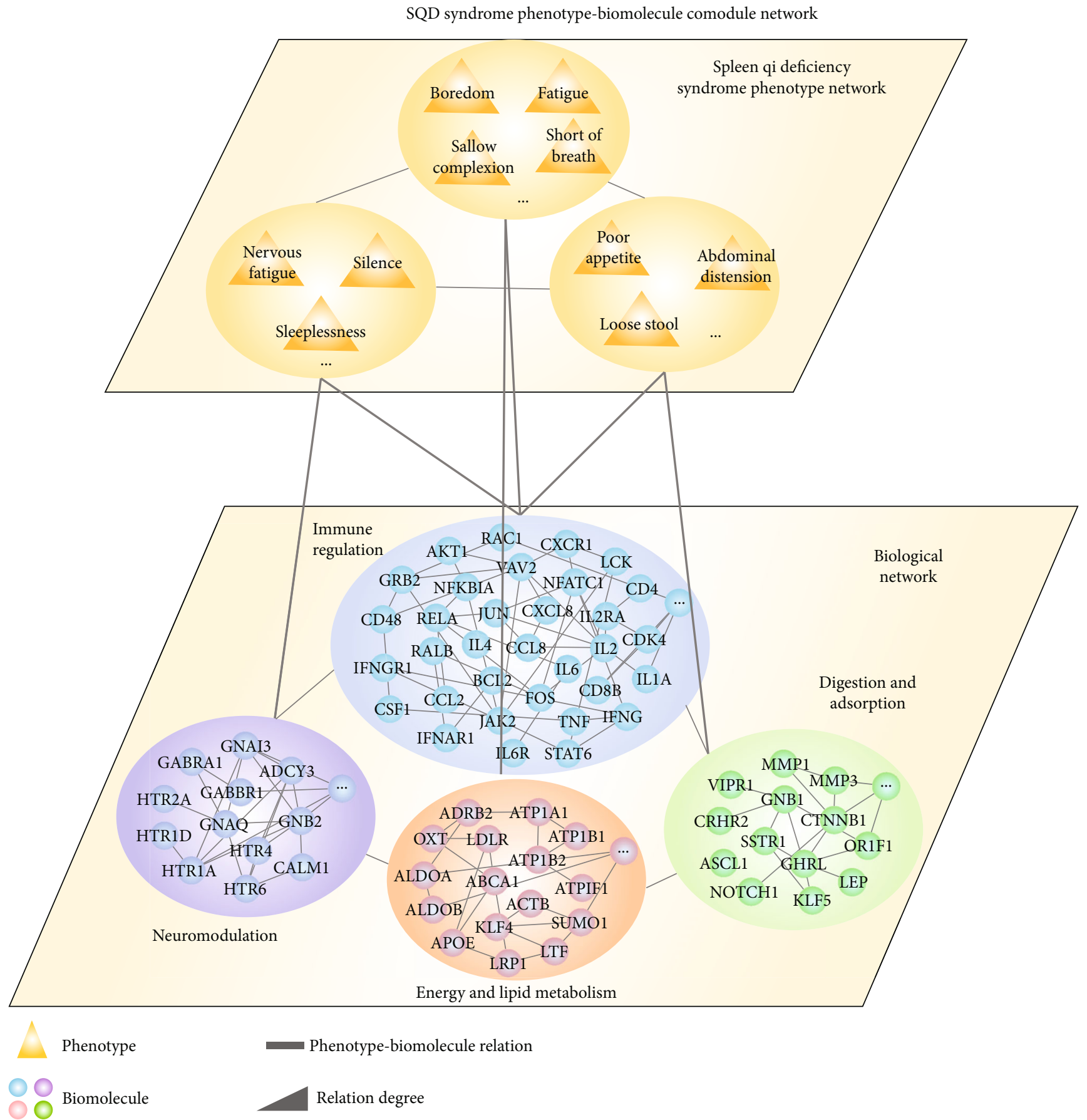

(a)

FIgUre 2: Continued. 
Biomolecular network underlying the SQD syndrome and immune functions

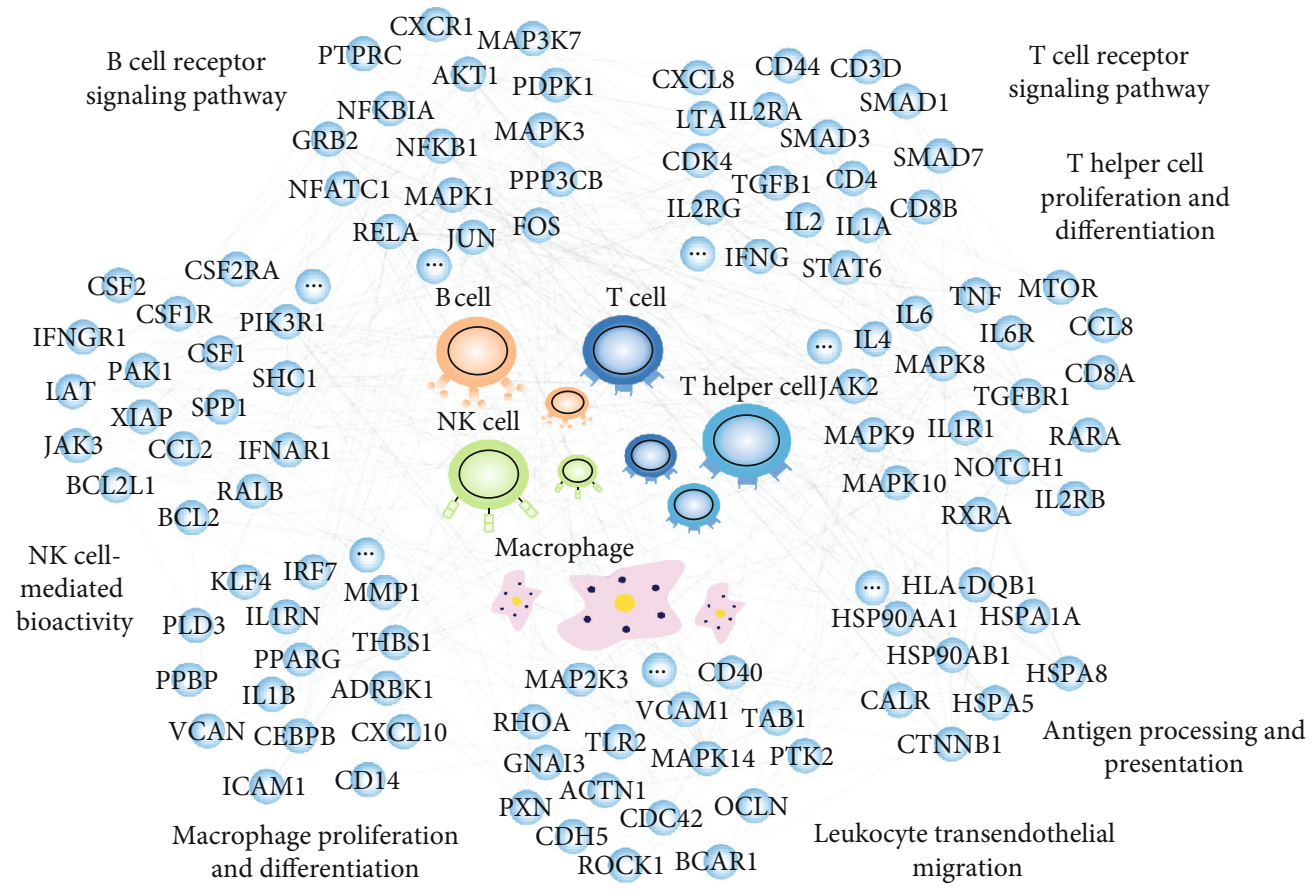

(b)

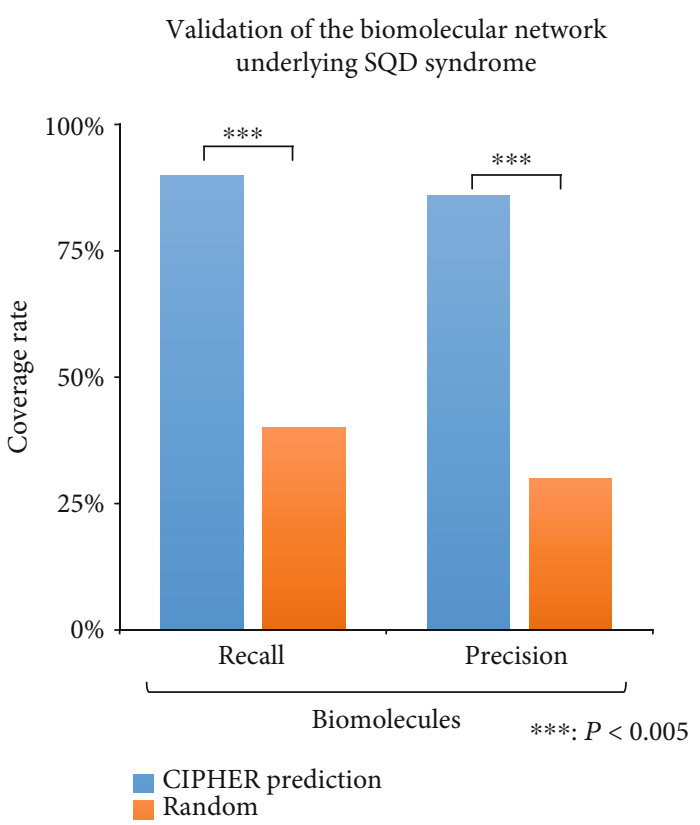

(c)

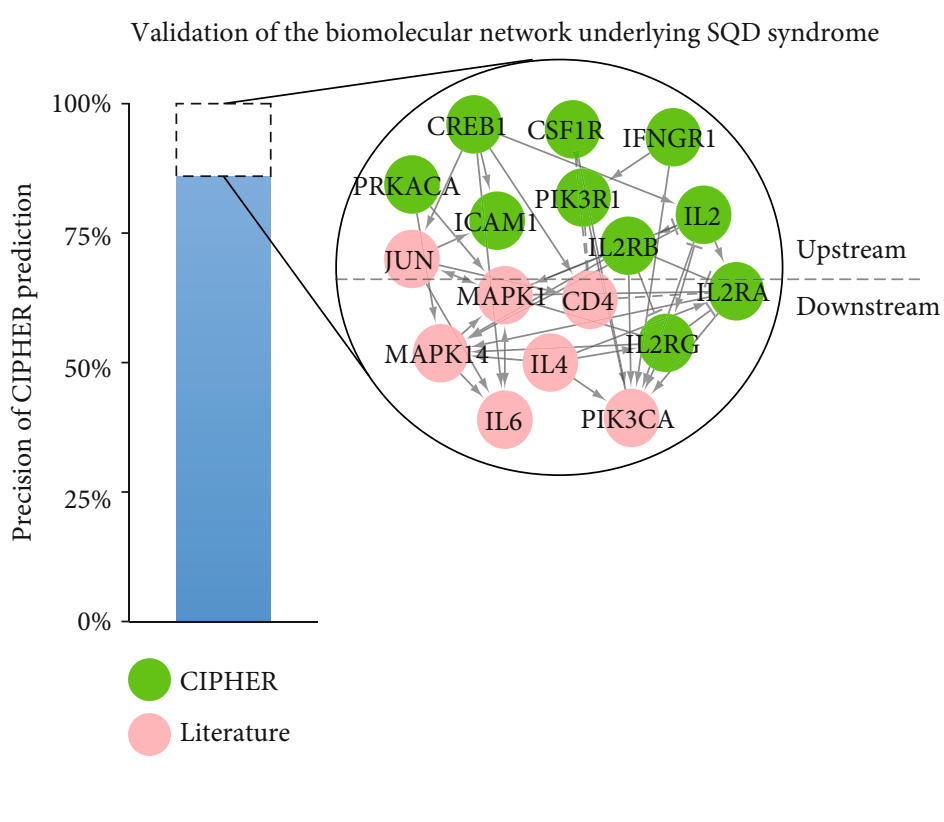

(d)

Figure 2: The biomolecular network underlying SQD syndrome was constructed based on clinical phenotypes and protein-protein interactions. (a) SQD syndrome phenotype-biomolecule comodule network. (b) SQD syndrome immune biomolecular network (biomolecules with significant enrichment in immune biological processes and pathways are marked, $P<0.05$ ). (c) Validation of the SQD syndrome biomolecular network. (d) The predicted biomolecules of SQD syndrome that are not reported in the literature are upstream or in the same pathway.

SQD syndrome (Figure 2(b)). The precision and recall rate of the predicted biomolecules related to SQD syndrome predicted by CIPHER were calculated for the validation via literature mining. The precision and recall rates of the predicted biomolecules related to SQD syndrome were significantly higher than those of the randomly selected biomolecules (Figure 2(c)). The top 20 nodes with the highest degrees in the immune biomolecular network of SQD syndrome and their biological functions are listed in Table 2. As shown in Figure 2(d), the predicted 
TABLE 2: Immune-related nodes in the SQD syndrome biomolecular network.

\begin{tabular}{lcc}
\hline $\begin{array}{l}\text { Top 20 } \\
\text { biomolecules }\end{array}$ & Degree & Biological process and pathway \\
\hline PIK3R1 & 29 & T cell receptor signaling pathway \\
MAPK14 & 27 & T cell receptor signaling pathway \\
LCK & 27 & Natural killer cell-mediated bioactivity \\
JUN & 26 & B cell receptor signaling pathway \\
GRB2 & 25 & T cell receptor signaling pathway \\
MAPK1 & 24 & T cell receptor signaling pathway \\
AKT1 & 24 & B cell receptor signaling pathway \\
MAPK8 & 24 & Helper T cell proliferation and \\
SMAD3 & 23 & T cell receptor signaling pathway \\
FYN & 23 & Natural killer cell-mediated bioactivity \\
RELA & 23 & B cell receptor signaling pathway \\
HSP90AA1 & 23 & Antigen processing and presentation \\
CTNNB1 & 21 & Antigen processing and presentation \\
SHC1 & 20 & Natural killer cell-mediated bioactivity \\
TGFBR1 & 20 & Helper T cell proliferation and \\
JAK2 & & differentiation \\
FOS & 17 & Helper T cell proliferation and \\
MAP3K7 & 17 & B cell receptor signaling pathway \\
NFKB1 & 17 & B cell receptor signaling pathway \\
MAPK3 & 17 & Leukocyte transendothelial migration \\
\hline
\end{tabular}

molecules related to SQD syndrome that are not referenced in the literature are upstream or in the same pathway. The SQD syndrome biomolecular network provides scientific support for further unveiling the biological basis of SQD syndrome.

3.2. Decreased Innate Immune Modules of the SQD Syndrome Biomolecular Network in Transcriptional Profiles of Chronic Gastritis Patients and IBS Patients. According to the holistic perspective of TCM, SQD syndrome is involved in both local and holistic disorders, which is closely related to many diseases. Literature mining was conducted to identify SQD syndrome-related diseases in the abstracts of the China National Knowledge Infrastructure (CNKI) database. Chronic gastritis and irritable bowel syndrome (IBS) are digestive diseases associated with SQD syndrome. We analyzed gene expression profiles in tissue samples from chronic atrophic gastritis (CAG) patients with the Cold syndrome (diagnosed as SSDC syndrome) or the Hot syndrome (diagnosed as SSDH syndrome) and IBS patients or normal individuals. SSDC syndrome and IBS are closely related to SQD syndrome which exhibits similar phenotypes (e.g., boredom, fatigue, lassitude, and loose stools). DEGs for chronic gastritis with the Cold syndrome and IBS patients in the SQD syndrome biomolecular network were involved in immune functions and response to oxidative stress (Figure 3(a)). DEG analysis revealed disorders in several immune functions and oxidative stress in chronic gastritis and irritable bowel syndrome (Figures 3(b) and 3(c)). In terms of immune functions, macrophage proliferation-related genes of the SQD syndrome biomolecular network were expressed at low levels in chronic gastritis patients with Cold syndrome by integrating the transcriptome data (Figures 3(d) and 3(e)). In the transcriptomic data of IBS patients, gene expression involved in NK cell activity was decreased in the network (Figures 3(f) and $3(\mathrm{~g})$ ). In terms of oxidative stress, the network node JAK2 mediates oxidative stress associated with the JAKSTAT signaling pathway and GSTT1 regulates the glutathione metabolic process to perform oxidative stress activity. By integrating the network analysis and transcriptional profile, we illustrated dysfunctions of the innate immune and oxidative stress modules in the SQD syndrome biomolecular network.

\subsection{Reduced Lymphocyte Activities of the SQD Syndrome} Biomolecular Network in Transcriptional Profiles of Patients with CFS and Patients with Hypertension. SQD syndrome is also a kind of the whole body's dysfunctions. According to the holistic perspective of TCM, SQD syndrome not only refers to local digestive disease but also contains the whole body's dysfunctions such as chronic fatigue syndrome (CFS) and hypertension. CFS and hypertension have some clinical manifestations of SQD syndrome [7, 37]. Therefore, in order to investigate the holistic biological basis of SQD syndrome, we analyzed gene expression profiles associated with CFS and hypertension for illustrating biological features of the SQD syndrome biomolecular network. The immunerelated DEGs in these diseases were significantly covered by the SQD syndrome immune biomolecular network $(P<0.05)$. Immune-related DEGs in CFS and hypertension were present in the SQD syndrome immune biomolecular network (Figure 4(a)). Analysis of differential gene expression revealed several immune dysfunctions in chronic fatigue syndrome (Figure 4(b)) and hypertension (Figure 4(c)). Some genes with decreased expression in CFS patients are significantly related to $\mathrm{T}$ cell function (Figure 5(a)). For example, low expression of NFATC1 regulates IFNG and other genes to reduce the adaptive immune response (Figure 5(b)). In addition, downregulated genes in hypertension patients are related to B cell function, as shown in Figure 5(c). Reductions in VAV3 and other genes in the B cell receptor signaling pathway lower Ig production (Figure 5(d)). The above results suggested a reduction in adaptive immune functions in SQD syndrome.

3.4. Network Pharmacology Analysis of Herbal Treatment for SQD Syndrome. SQD syndrome exhibits its specific phenotypes and is closely linked to herbs for reinforcing spleen qi. Therefore, SQD syndrome is not only related to phenotypes but also associated with herbal treatment. Five herbs were commonly used for phenotypes associated with SQD syndrome in the CNKI database, that is, herbs for invigorating spleen and tonifying spleen qi such as Rhizoma Atractylodis Macrocephalae, Radix Astragali, Radix Codonopsis Pilosulae, Radix Ginseng, and Rhizoma Dioscoreae. These herbs are also used to treat SQD syndrome-related diseases [11, 38-40]. 
Chronic gastritis and IBS differentially expressed genes in the SQD syndrome biomolecular network

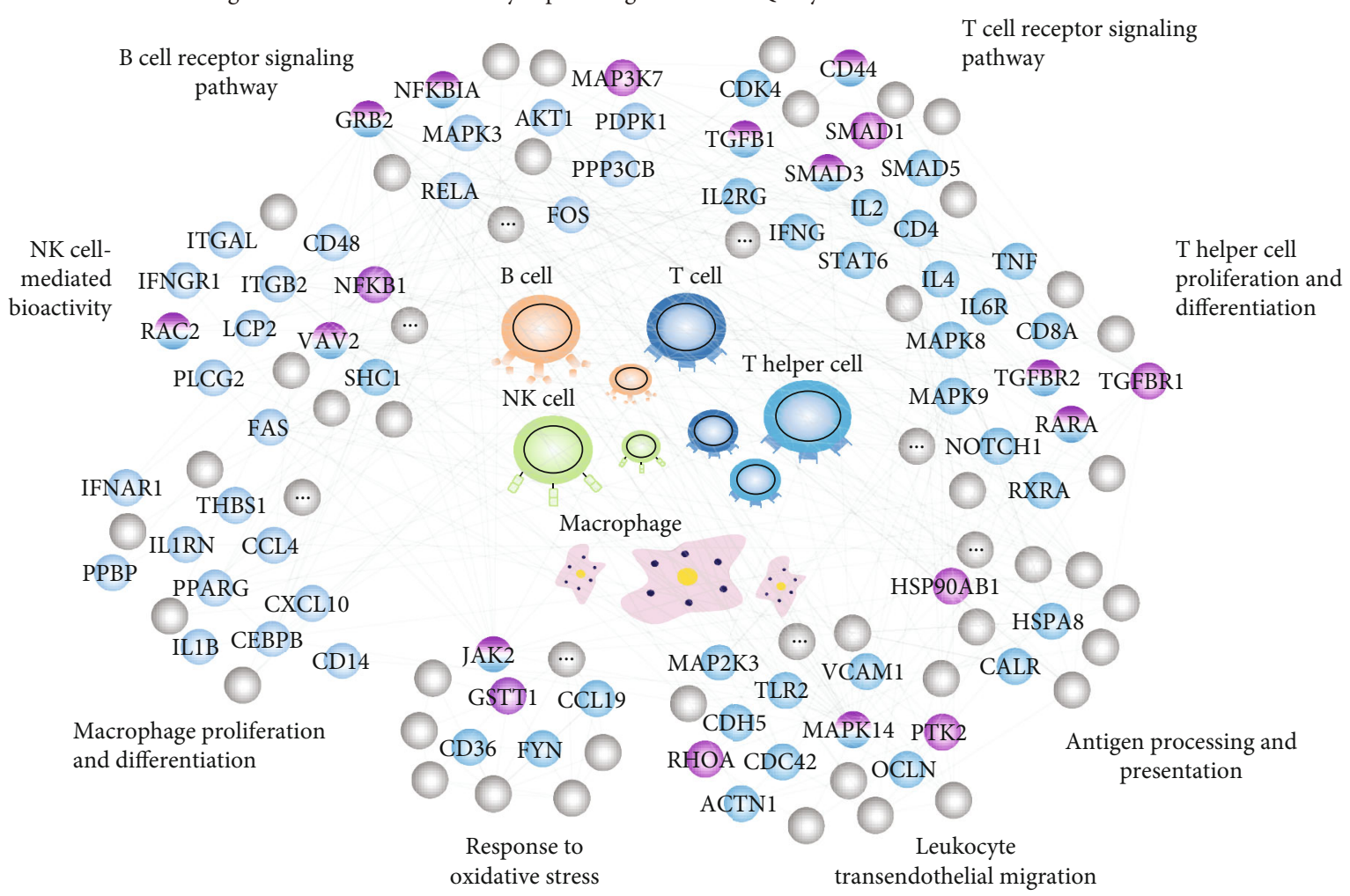

(a)

Gene expression profile of chronic gastritis patients with SSDC syndrome or SSDH syndrome

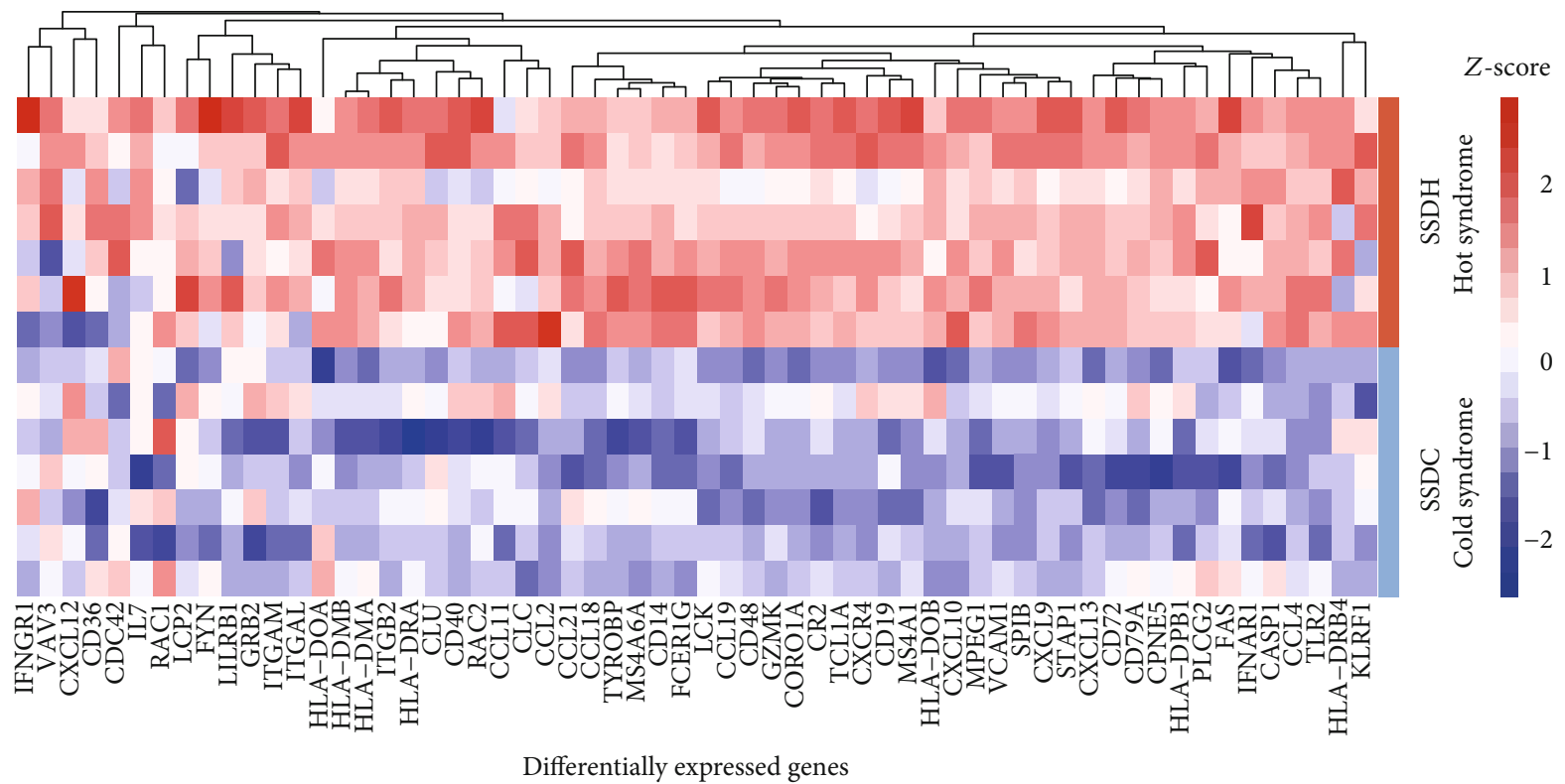

(b)

Figure 3: Continued. 
Gene expression profile of normal and IBS patients

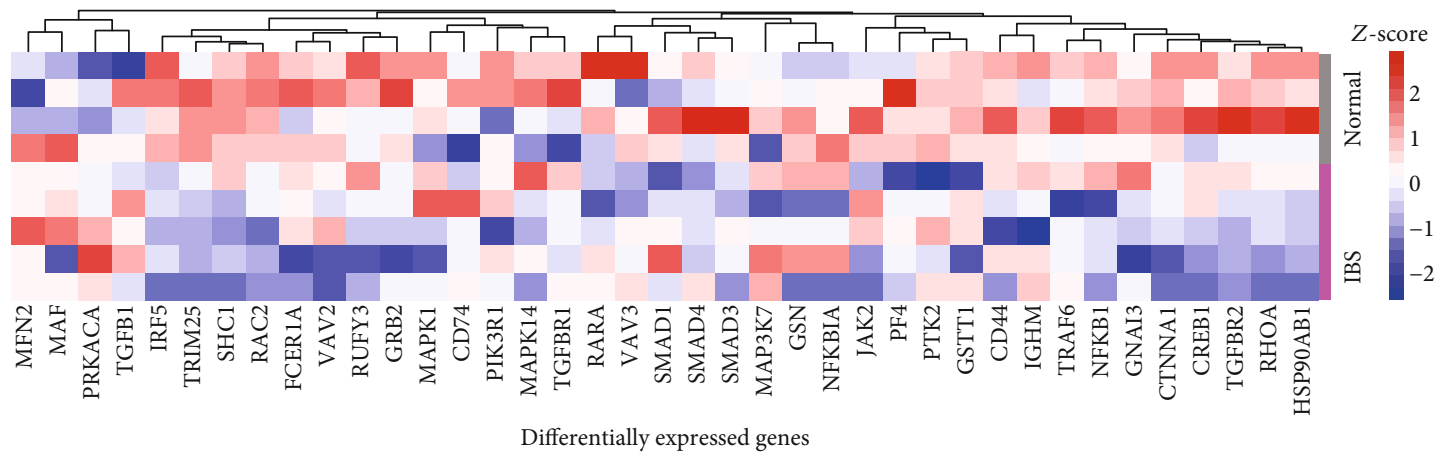

(c)

Gene expression in chronic gastritis with SSDC syndrome or SSDH syndrome patients

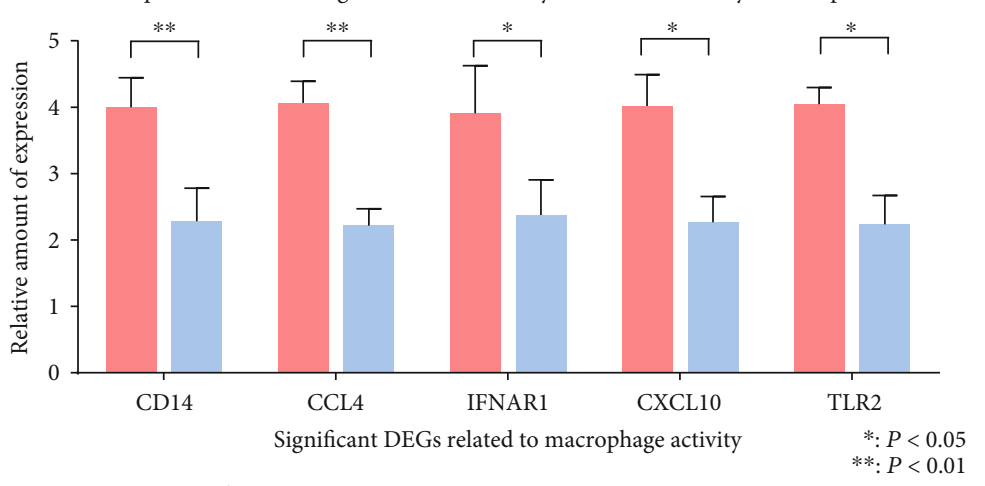

- SSDH syndrome patients

- SSDC syndrome patients

(d)
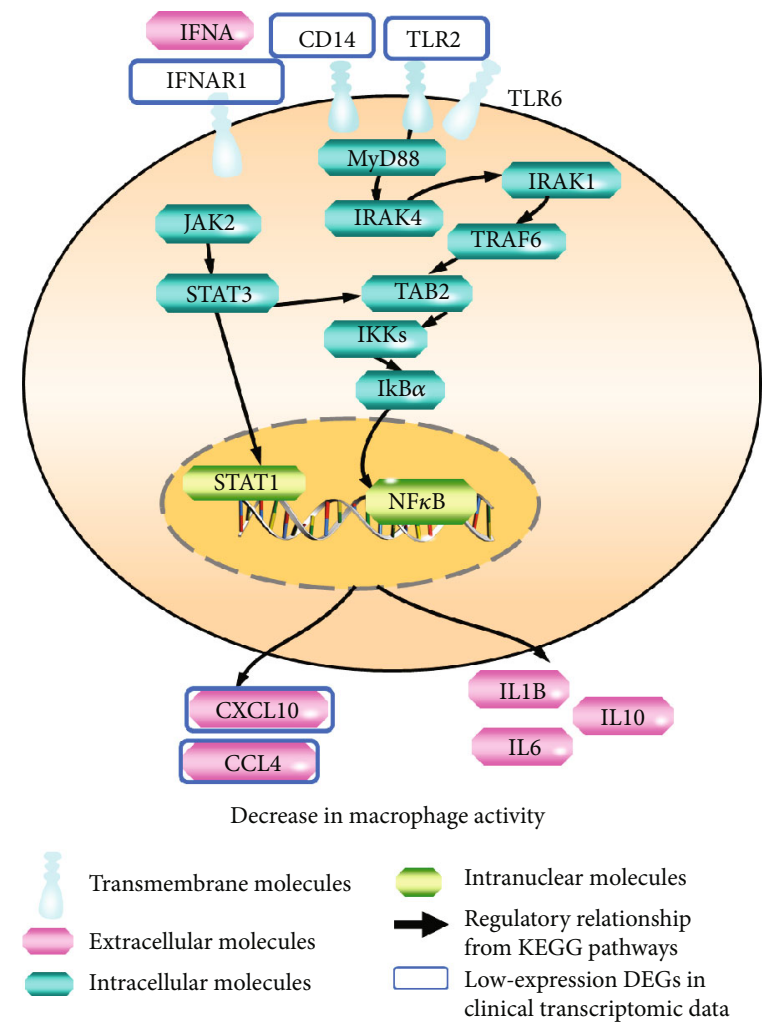

(e)

Figure 3: Continued. 
Gene expression in normal and IBS patients

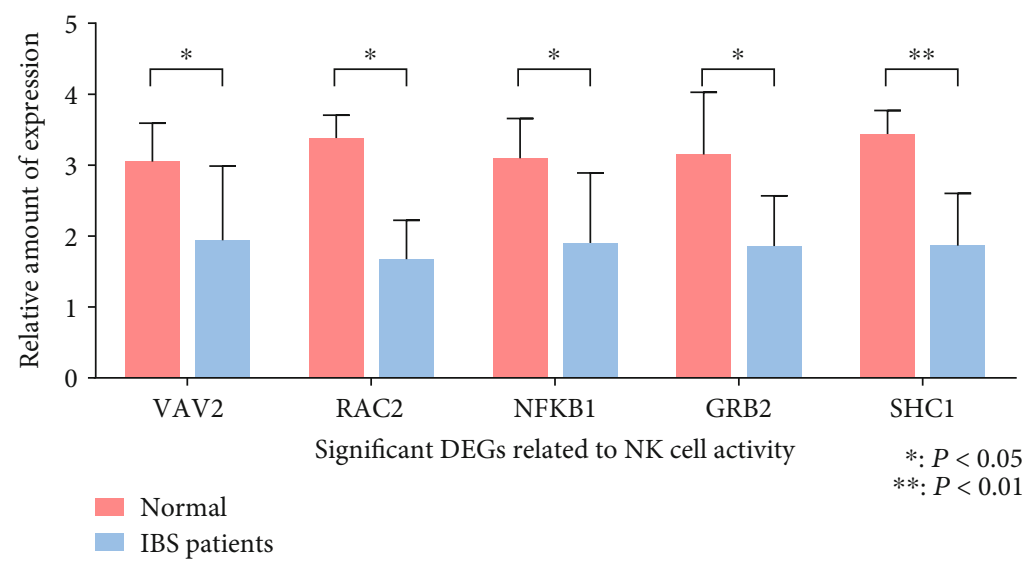

(f)

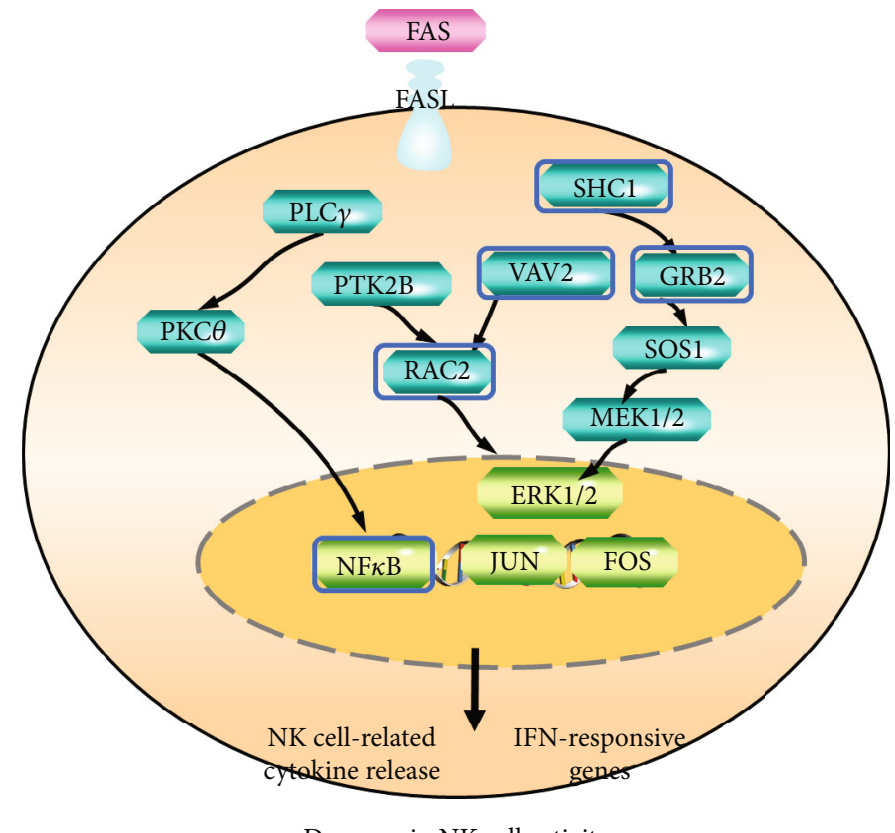

Decrease in NK cell activity
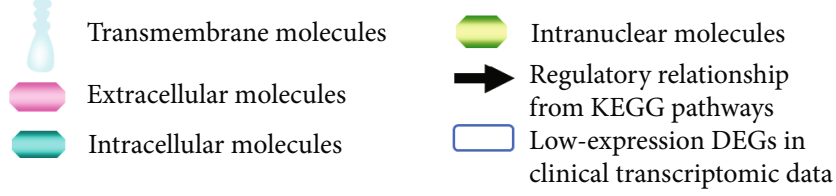

(g)

FIGURE 3: The immune molecular network of SQD syndrome covers the DEGs in immune function in chronic gastritis patients and IBS patients. (a) DEGs in chronic gastritis patients with SSDC syndrome and DEGs in IBS patients in the SQD syndrome biomolecular network. (b) The expression of the selected genes in patients with chronic gastritis with SSDC syndrome or SSDH syndrome. (c) The expression of the selected genes in normal individuals and IBS patients. (d) Gene expression related to macrophages in chronic gastritis with SSDC syndrome. (e) DEGs in chronic gastritis with SSDC syndrome in the immune molecular network of SQD syndrome that reduce macrophage function. ${ }^{*} P<0.05,{ }^{* *} P<0.01$ : compared with chronic gastritis patients with SSDH syndrome. (f) Gene expression related to NK cell-mediated bioactivity in IBS patients. (g) DEGs in IBS patients that regulate the NK cell pathway in the SQD syndrome biomolecular network of SQD syndrome. ${ }^{*} P<0.05,{ }^{* *} P<0.01$ : compared with the normal group.

Herbal ingredients tend to bind multiple targets. Thus, we sought to identify the targets regulated by the herbal ingredients and to understand the relationships between the pre- dicted targets $[18,41]$. We validated the reliability of the predicted targets by literature mining on herbal ingredients for SQD syndrome with greater than 100 literature records. 
CFS and hypertension differentially expressed genes in SQD syndrome immune biomolecular network

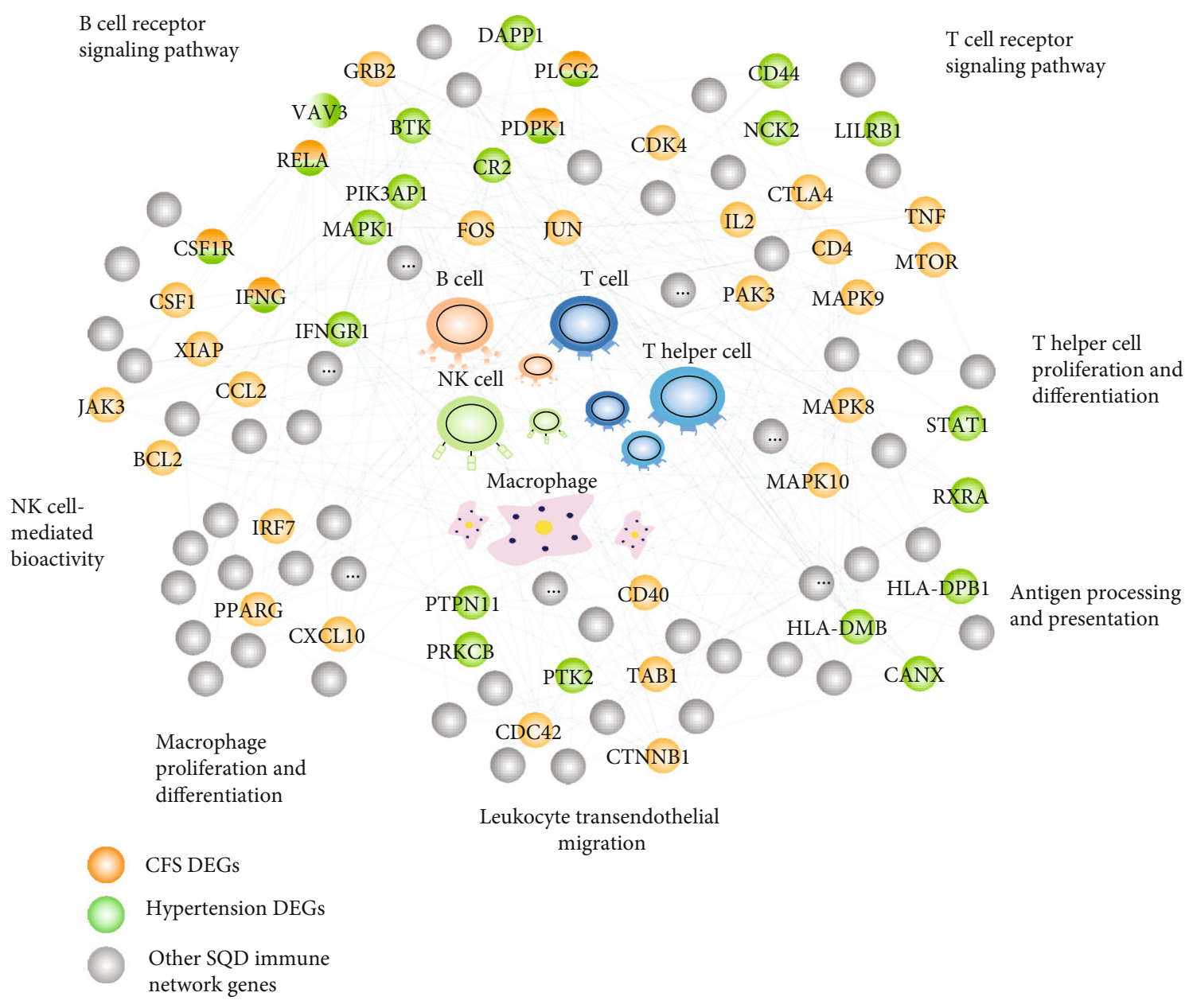

(a)

Gene expression profile of normal and CFS patients

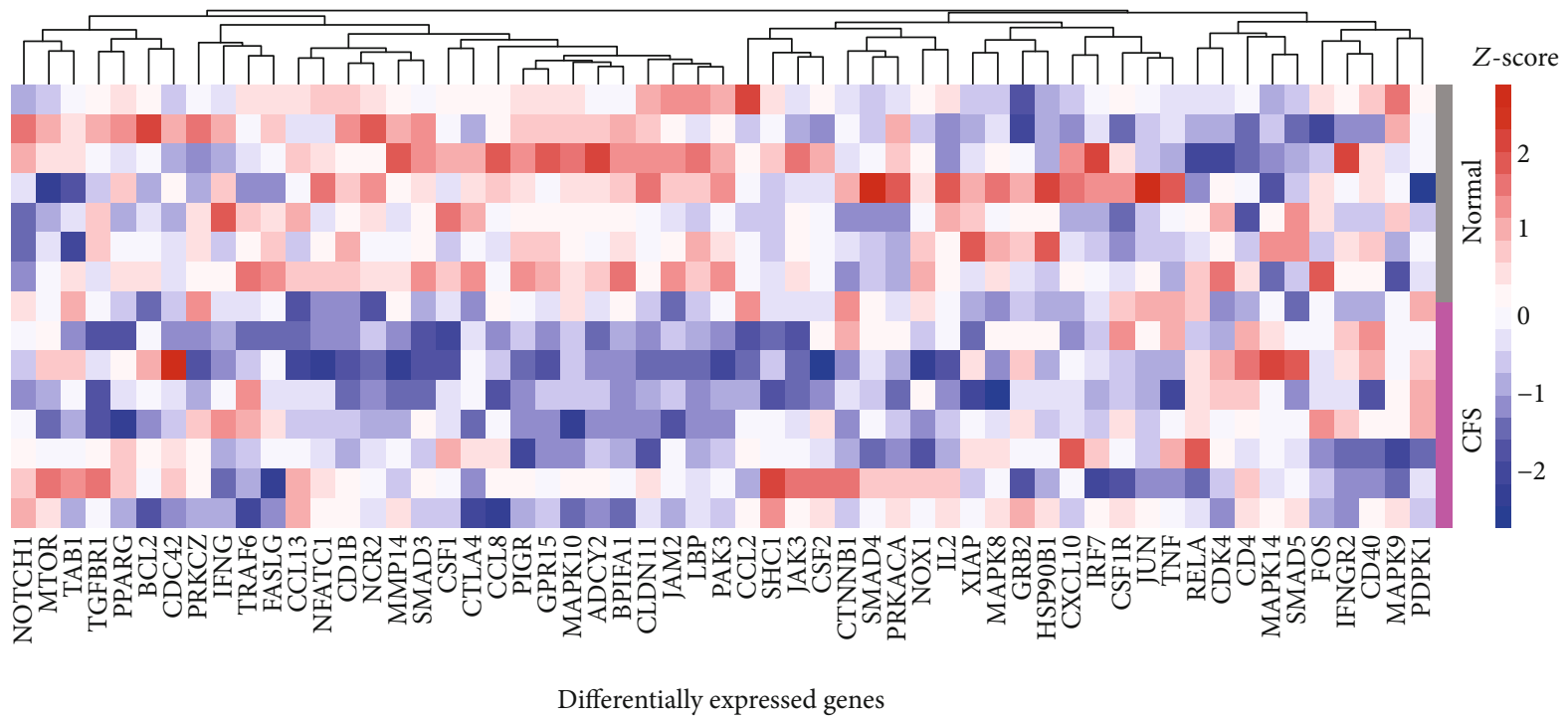

(b)

Figure 4: Continued. 
Gene expression profile of normal and hypertension patients

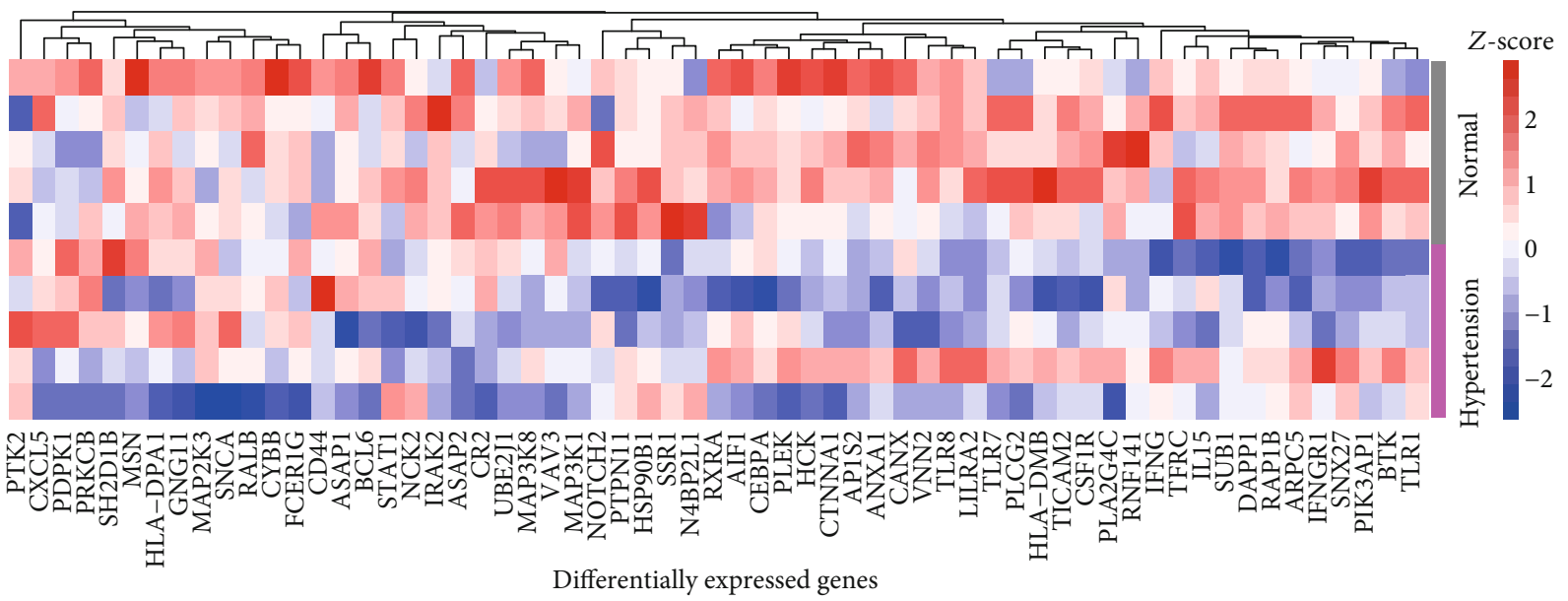

(c)

FIGURE 4: Immune biomolecular network of SQD syndrome contains immune function of SQD syndrome-related diseases. (a) DEGs in CFS or hypertension in the immune molecular network of SQD syndrome. (b) The expression of the selected genes in normal individuals and patients with CFS. (c) The expression of the selected genes in normal individuals and patients with hypertension.

As shown in Figure 6(a), greater than $70 \%$ of the biomolecules related to representative herbal ingredients with literature evidence were linked to potential targets via direct mapping or indirect connections, such as PPIs or signaling pathways. The results show that the predicted targets can help to describe the mechanisms of these herbs for SQD syndrome.

The SQD syndrome immune biomolecular network can be applied to distinguish herbs for SQD syndrome and those for other syndromes. Here, five herbs with no literature evidence for treating SQD syndrome in the CNKI database (Radix Isatidis, Cassia Seed, Swertia, Herba Lysimachia, and Folium Isatidis) were selected as herbs for syndromes other than SQD syndrome (Figure 6(b)). The biomolecules related to herbs for SQD syndrome covered the network (Figure 6(c)). Moreover, the topological relationship between the predicted targets of herbs for SQD syndrome and the network was measured by NIMS, a network-based topological analysis method [42]. The analysis results revealed a significant correlation between the predicted targets of herbs for SQD syndrome and the immune biomolecular network (Figure 6(d)). As shown in Supplementary Table S2, the target lists of herbal ingredients for SQD syndrome are covered in the SQD syndrome immune biomolecular network. This network uncovers the immune regulation effects of herbs for SQD syndrome.

3.5. Experimental Validation of Herbal Ingredients for Immune Modules of the SQD Syndrome Biomolecular Network. According to the traditional efficacy of Chinese medicine in the Compendium of Materia Medica, Rhizoma Atractylodis Macrocephalae is used for invigorating the spleen to eliminate dampness and Radix Astragali is the most common herb for tonifying spleen qi. Based on the traditional efficacy of Chinese medicine and literature mining for treating SQD syndrome, Radix Astragali is suitable to reg- ulate the SQD syndrome-related immune functions [43]. Astragalus saponin ingredients and astragalus polysaccharides are the representative ingredients of an herb for SQD syndrome, Radix Astragali. The predicted targets of these ingredients were related to the SQD syndrome biomolecular network (Figure $7(\mathrm{a})$ ). In order to confirm that the immune regulation of the representative ingredients of herbs for SQD syndrome is revealed in the SQD syndrome biomolecular network, we find experimental evidence for immune regulation of some ingredients in literature and further conducted pharmacological experiments. Experimental results illustrated that astragaloside saponin ingredients and astragalus polysaccharide have immunoregulation of pharmacological activities in vivo and in vitro. For example, astragaloside saponin ingredients and astragalus polysaccharides have strong promoting effects on the phagocytosis of macrophages [44]. Astragaloside IV could increase T and B lymphocyte proliferation and antibody production [45]. Astragalus saponin ingredients (astragaloside I, astragaloside II, and astragaloside IV) and astragalus polysaccharides promote the proliferation of macrophages (Figure 7(b)). Experimental results demonstrated that astragaloside IV alone could significantly enhance the proliferation of splenic lymphocytes with or without ConA or LPS stimulation and the effects were stronger than those of the vehicle control (Figure 7(c)). The experimental results further demonstrated that astragalus saponin ingredients and astragalus polysaccharides enhance immune regulation by macrophage and lymphocyte modules in the SQD syndrome biomolecular network.

\section{Discussion}

Traditional Chinese medicine states that "pathogenic-qi cannot invade the body if "health-qi" remains strong" in Plain Questions about Huangdi Neijing. "Spleen qi" is an important 

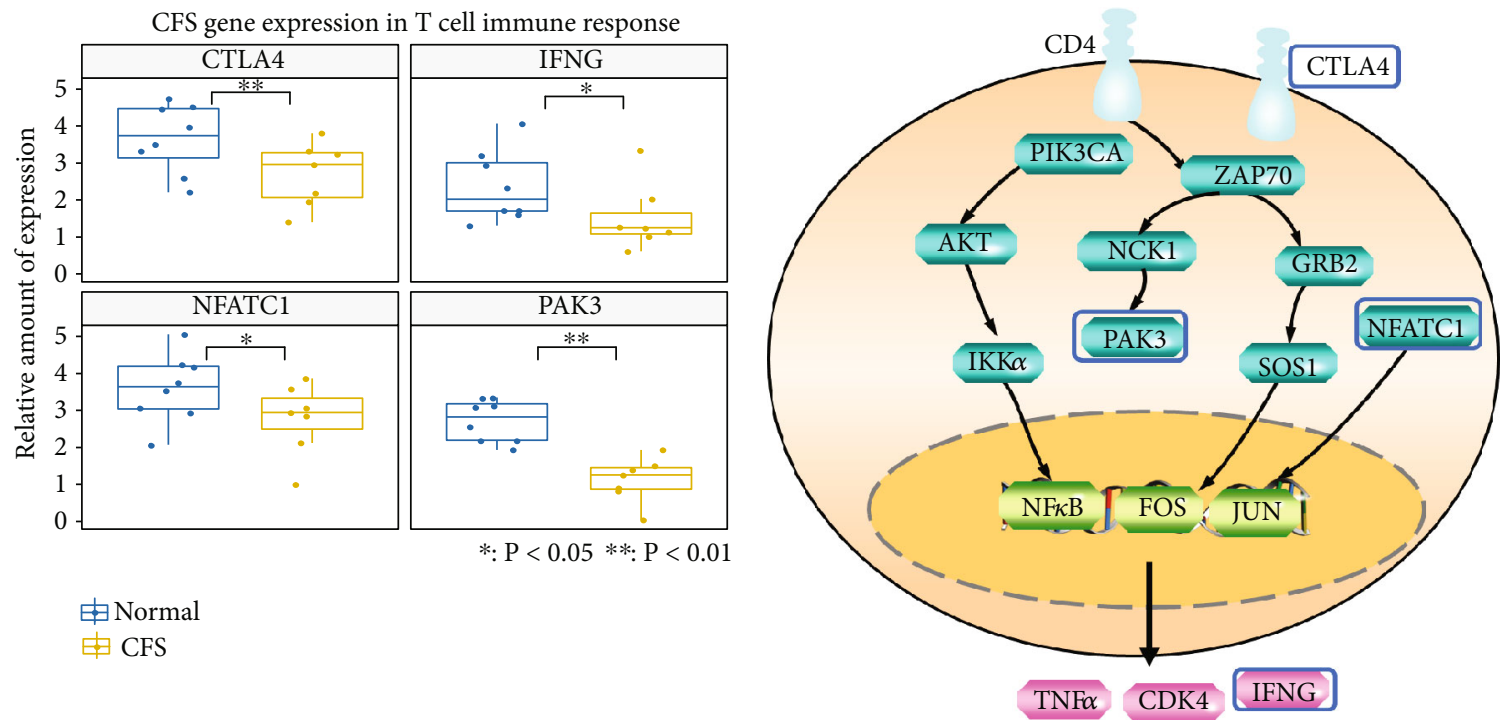

Decrease in $\mathrm{T}$ cell immune response

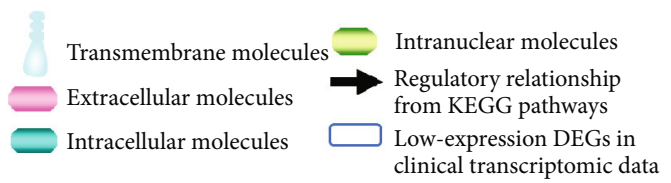

(a)

(b)

Hypertension gene expression in B cell immune response
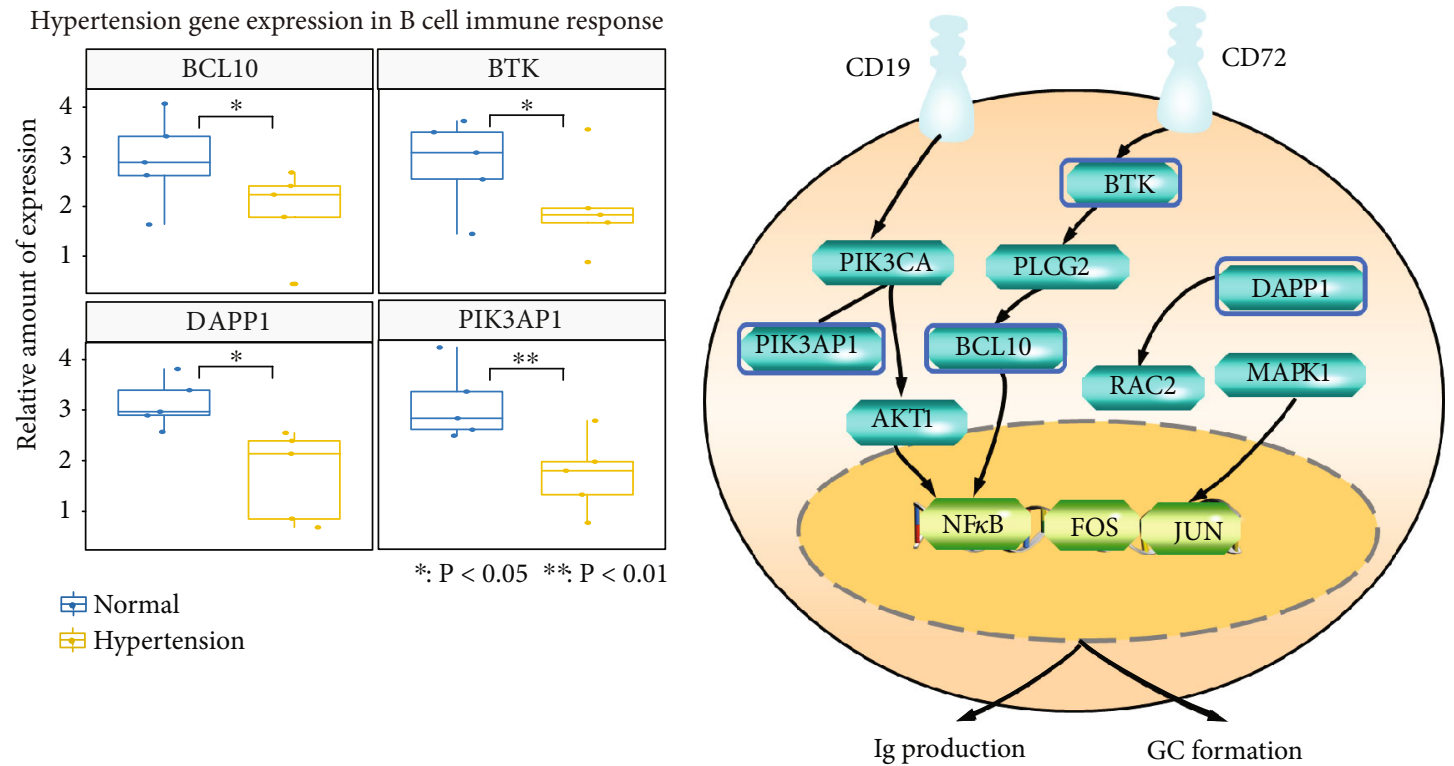

Decrease in B cellimmuneresponse

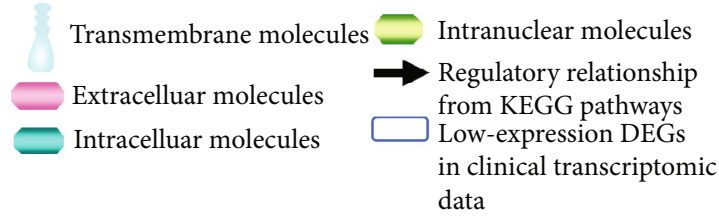

(c)

(d)

FIGURE 5: (a) Selected gene expression in normal individuals and CFS patients. (b) DEGs in CFS in the immune molecular network of SQD syndrome that reduced $\mathrm{T}$ cell immune function. (c) Selected gene expression in normal individuals and hypertension patients. (d) DEGs in hypertension in the immune molecular network of SQD syndrome that reduced B cell immune function. ${ }^{*} P<0.05,{ }^{* *} P<0.01$ : compared with the normal group. 


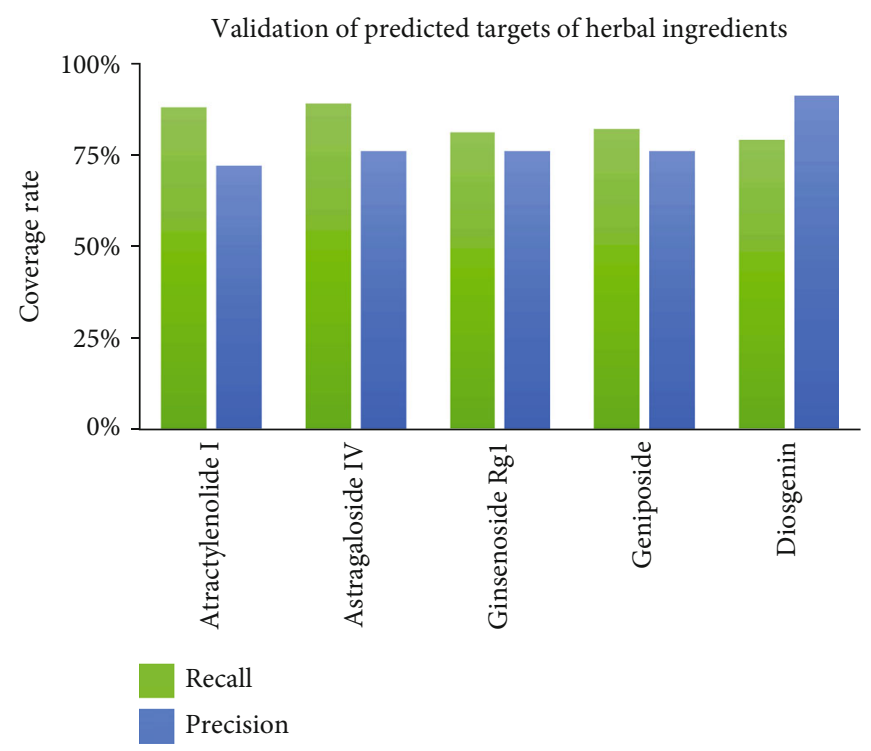

(a)
Comparison of predicted targets of herbs for SQD syndrome and herbs for other syndrome on the network

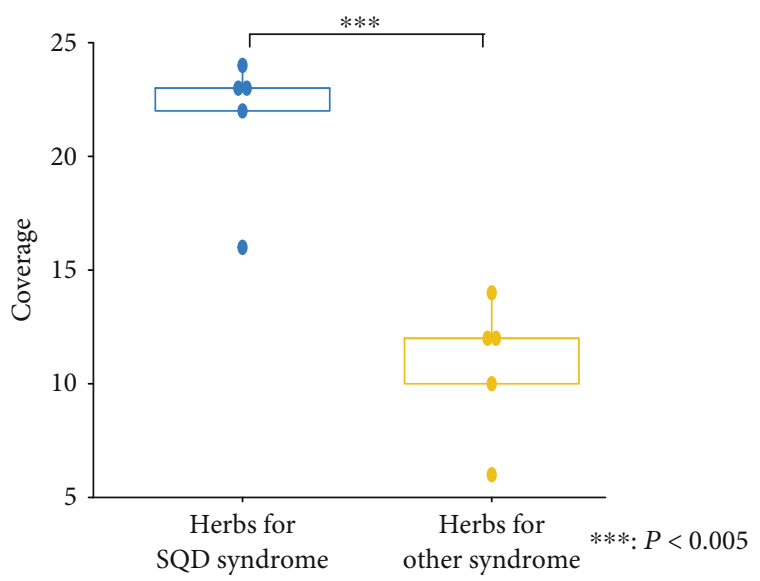

Immune biomolecular network of SQD syndrome regulated by herbs

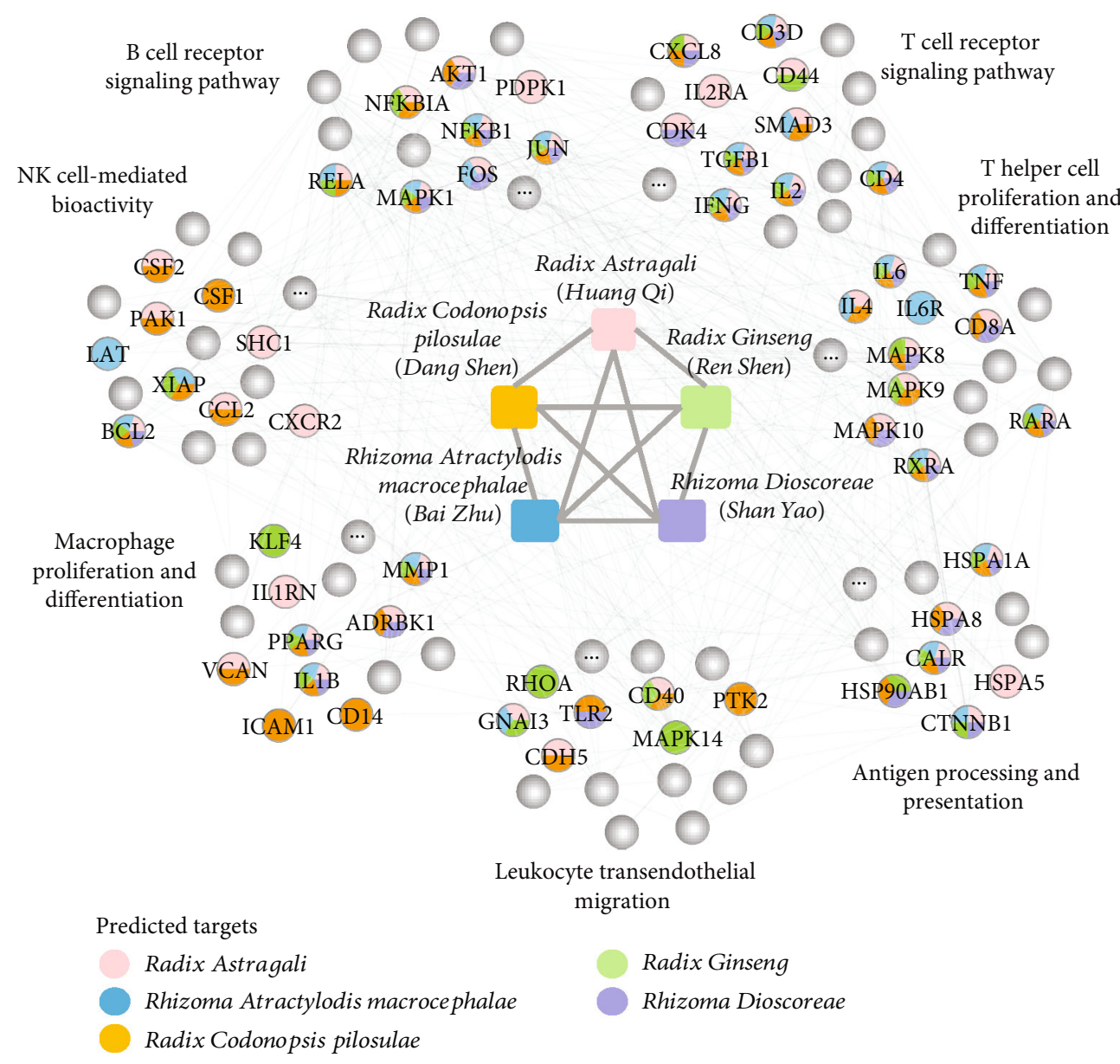

(c)

Figure 6: Continued. 


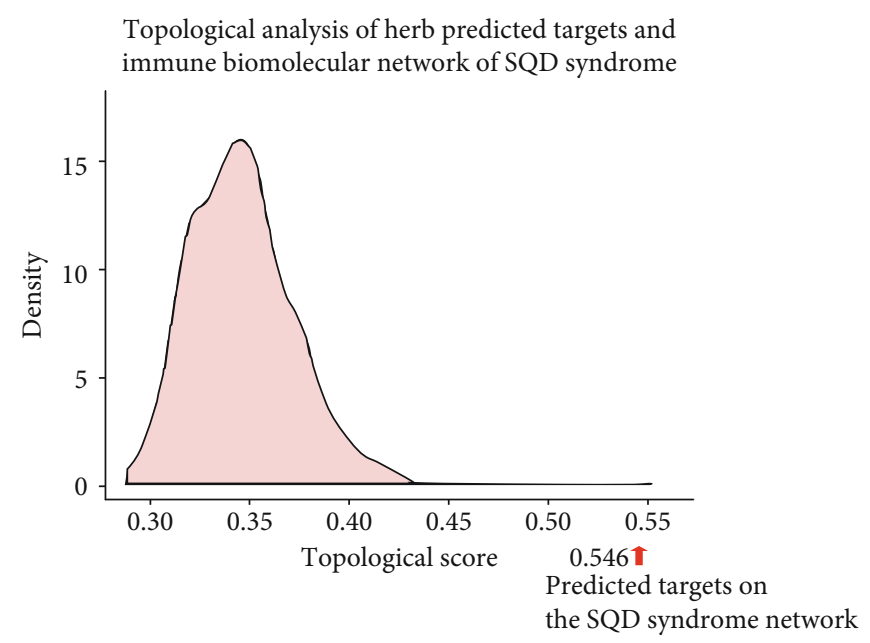

(d)

FIGURE 6: The immune biomolecular network of SQD syndrome uncovers the mechanisms of action of commonly used herbs for SQD syndrome. (a) Validation of the drugCIPHER-predicted targets of compounds in herbs for SQD syndrome. (b) The predicted targets of herbs for SQD syndrome were significantly enriched in the immune biomolecular network of SQD syndrome compared to herbs for other syndromes. (c) The SQD syndrome immune molecular network covers the predicted targets and the features of herbs for SQD syndrome. (d) The predicted targets of herbs for SQD syndrome are closely related to the immune biomolecular network of SQD syndrome in the topological structure compared to the random situation.

category of "health qi." The biological basis of SQD syndrome is unclear, and the associated mechanisms of disease-syndrome-herb are not known. This situation has caused certain difficulties for clinical diagnosis and herbal treatment. Rats treated with reserpine, which exhibit similar signs to SQD syndrome, exhibit neuro-endocrine-immune disorders [46]. In hypertension, a deficiency in macrophage function and B cell dysfunction induced blood pressure elevation and vascular injury [47]. As shown in our results, SQD syndrome-related biological functions are also associated with oxidative stress. The antioxidant module of the SQD syndrome biomolecular network decreases in transcriptional profiles of patients with chronic gastritis, which leads to oxidative stress. Oxidative stress could reduce the activity of NK cells and T lymphocytes [48]. Increased oxidative stress damage and insufficient immune regulation lead to cellular dysfunction causing aging [49].

Given that disease is a multifactorial consequence, the corresponding drug combinational therapy regulates multiple targets to produce therapeutic effects. SQD syndrome is related to the reduction in immune functions and antioxidant activity [50]. The molecular mechanisms of herbal formula in treating diseases and syndromes are closely related. For example, we used a heterogeneous biological network of the association of disease-SQD syndrome-herb to reveal relevant molecular mechanisms. Radix Astragali treats SQD syndrome-related diseases by improving immune function and inhibiting oxidative stress [51, 52]. The experimental results also demonstrated that astragaloside I, astragaloside II, astragaloside IV, and astragalus polysaccharides may increase the proliferation ability of the macrophage and lymphocyte in the SQD syndrome bimolecular network. The ingredients in Radix Ginseng regulate a portion of the biological functions including NK cell activity and antioxidant activity in the SQD syndrome bimolecular network $[53,54]$.
Research on SQD syndrome by systems biology approaches, including metagenomics, has been increasingly emerging [55]. Network pharmacology studies have investigated the biological basis of TCM syndrome from the perspective of biomolecular networks [18]. This is a promising approach that is consistent with the features of TCM syndrome. The efficacy of the SQD syndrome immune network can be attributed to the following aspects. First, the integration of phenotypes and biomolecular networks helps identify the biological basis of SQD syndrome, which could contribute to the development of effective treatments. Second, the biomolecular network contains the biological features of differentially expressed genes in SQD syndrome-related diseases and herbal treatment. However, there are some limitations of the present study. The identified immune functions in the SQD syndrome-related network need to be further verified in large clinical samples. Further investigation should be conducted to detect different tissues in order to analyze the tissue specificity of SQD syndrome. Besides, the herbal ingredients for SQD syndrome used in the present study are still incomplete. Other herbs for SQD syndrome and more ingredients in vivo and in vitro need to be further collected.

\section{Conclusion}

In summary, this study proposed a novel network pharmacology strategy to predict the SQD syndrome biomolecular network. The clinical transcriptomic data and pharmacological experiments further evaluated and validated the biological features of the SQD syndrome-related network. TCM is a type of personalized medicine with long-term clinical practice experience. This study used SQD syndrome as an example to uncover the biological basis of SQD syndrome from the network pharmacology perspective, thereby providing a way 
SQD syndrome immune biomolecular network regulated by astragalus saponins and astragalus polysaccharide

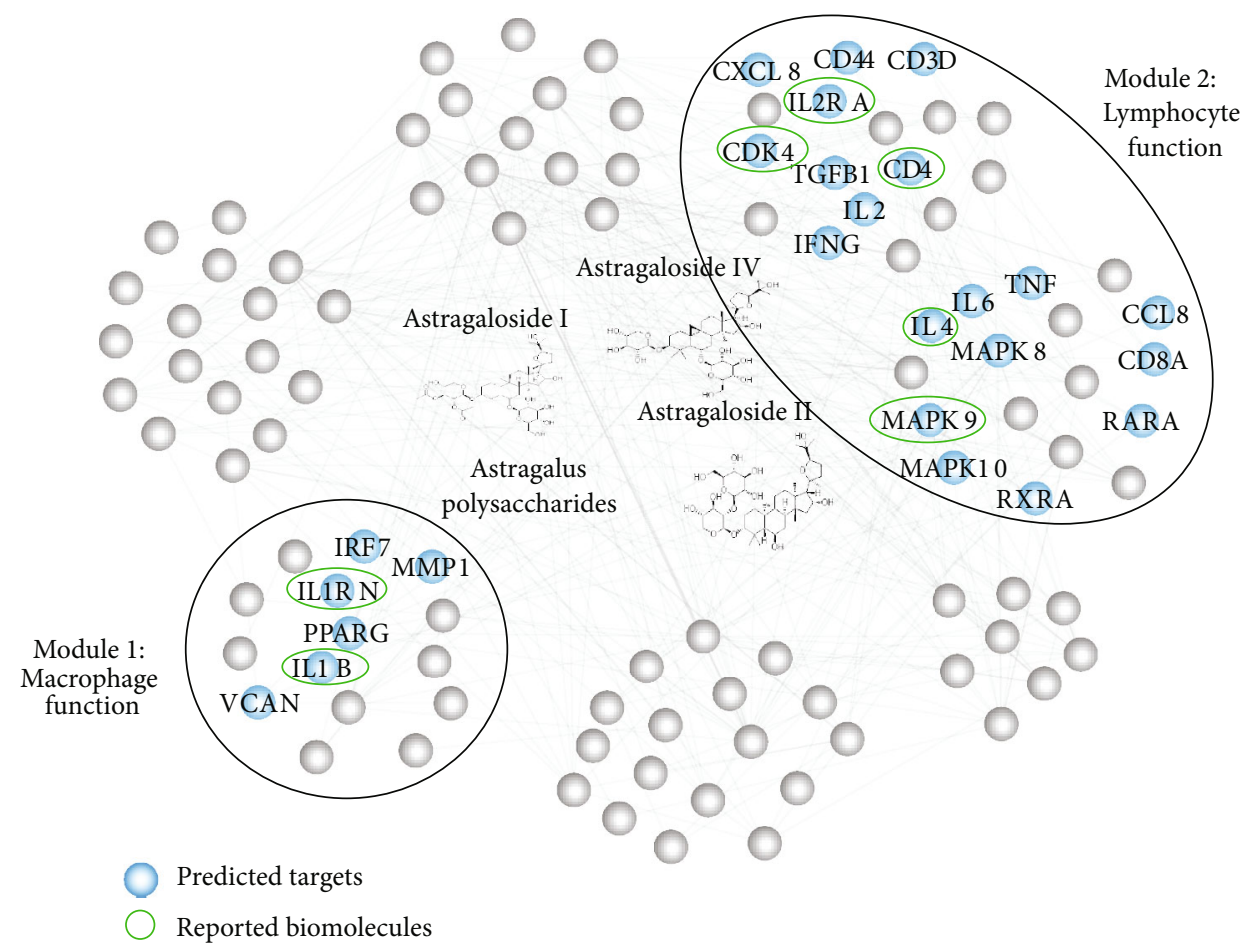

(a)

SQD syndrome-related macrophage function module promoted by Radix Astragali ingredients

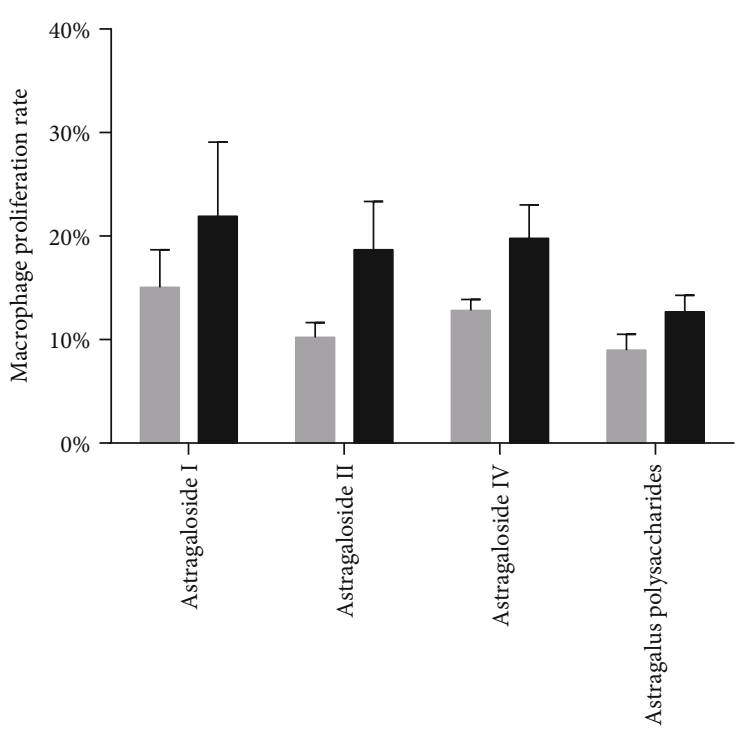

$5 \mu \mathrm{mol} / \mathrm{L}$

$50 \mu \mathrm{mol} / \mathrm{L}$

(b)
SQD syndrome-related lymphocyte function promoted by astragaloside IV

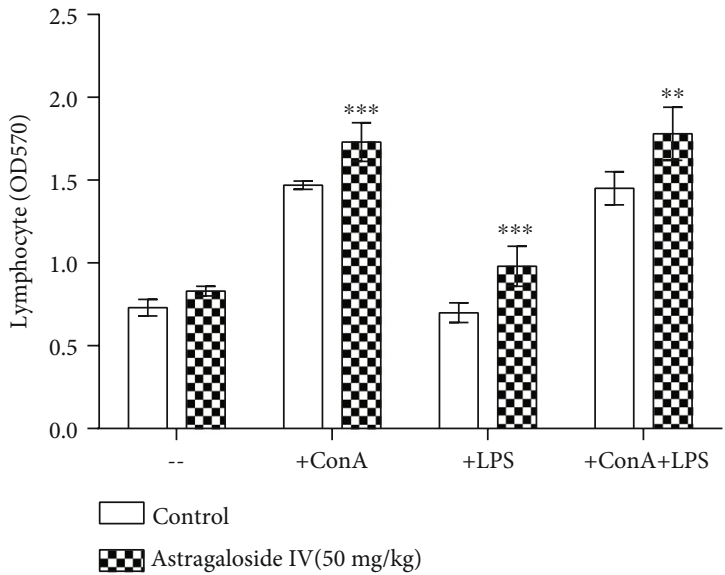

FIGURE 7: Ingredients in herbs for SQD syndrome may exert immune-enhancing activity related to the immune biomolecular network of SQD syndrome. (a) The predicted targets of the representative ingredients in Radix Astragali may regulate immune function in the macrophage and lymphocyte function modules in the SQD syndrome biomolecular network. (b) Representative ingredients in Radix Astragali improve macrophage proliferation. (c) Astragaloside IV promotes the proliferation of spleen lymphocytes. ${ }^{* *} P<0.01,{ }^{* * *} P<$ 0.005: compared with the control group. 
for the precision diagnosis and treatment of SQD syndromerelated diseases such as chronic gastritis and hypertension. Based on the network pharmacology approach, this study not only reveals parts of the biological basis of SQD syndrome but also provides a novel insight for exploring the mechanisms of SQD syndrome-related diseases.

\section{Abbreviations \\ TCM: Traditional Chinese medicine \\ SQD: $\quad$ Spleen qi deficiency \\ SSDC: Spleen stomach deficiency Cold \\ SSDH: Spleen stomach dampness Hot \\ IBS: Irritable bowel syndrome \\ CFS: Chronic fatigue syndrome \\ DEGs: Differentially expressed genes \\ wQED: Weighted quantitative estimate of druglikeness \\ DMSO: Dimethyl sulfoxide.}

\section{Data Availability}

Further information and requests for data may be directed to and will be fulfilled by the lead contact Shao Li (shaoli@tsinghua.edu.cn) on a reasonable request.

\section{Conflicts of Interest}

The authors declare no conflict of interest.

\section{Authors' Contributions}

S.L. conceived and supervised the study. X.W., M.W., and J.H.Z. performed the network pharmacology analysis. M.W., X.X.L., M.H.H., and Y.L. performed the experiments and analyzed the data. All authors discussed the results and wrote the manuscript. X. W. and M. W. contributed equally to this work.

\section{Acknowledgments}

This study was supported in part by the National Natural Science Foundation of China (grant numbers 81630103, 91729301, and 81225025).

\section{Supplementary Materials}

Table S1: clinical phenotypes of spleen qi deficiency syndrome. Table S2: SQD syndrome biomolecular network regulated by partial ingredients in herbs for reinforcing spleen qi. (Supplementary Materials)

\section{References}

[1] S. Zhang, L. Hu, and R. Li, "Expert consensus on tcm diagnosis and treatment of spleen deficiency syndrome," Journal of Traditional Chinese Medicine, vol. 58, no. 17, pp. 1525-1530, 2017.

[2] J. Lin, J. Hu, and B. Liu, "Research on conceptual framework of pro scale in spleen-qi deficiency syndrome," Tianjin Journal of Traditional Chinese Medicine, vol. 30, no. 5, pp. 277-281, 2013.
[3] X. F. Zheng, J. S. Tian, P. Liu, J. Xing, and X. M. Qin, "Analysis of the restorative effect of $B u$-zhong-yi-qi-tang in the spleen-qi deficiency rat model using ${ }^{1} \mathrm{H}$-NMR-based metabonomics," Journal of Ethnopharmacology, vol. 151, no. 2, pp. 912-920, 2014.

[4] S. Li, Z. Q. Zhang, L. J. Wu, X. G. Zhang, Y. D. Li, and Y. Y. Wang, "Understanding ZHENG in traditional Chinese medicine in the context of neuro-endocrine-immune network," IET Systems Biology, vol. 1, no. 1, pp. 51-60, 2007.

[5] T. Ma, C. Tan, H. Zhang, M. Wang, W. Ding, and S. Li, "Bridging the gap between traditional Chinese medicine and systems biology: the connection of cold syndrome and NEI network," Molecular BioSystems, vol. 6, no. 4, pp. 613-619, 2010.

[6] R. Li, T. Ma, J. Gu, X. Liang, and S. Li, "Imbalanced network biomarkers for traditional Chinese medicine Syndrome in gastritis patients," Scientific Reports, vol. 3, no. article 1543, 2013.

[7] Y. H. Kuo, W. J. Tsai, S. H. Loke, T. S. Wu, and W. F. Chiou, "Astragalus membranaceus flavonoids (AMF) ameliorate chronic fatigue syndrome induced by food intake restriction plus forced swimming," Journal of Ethnopharmacology, vol. 122, no. 1, pp. 28-34, 2009.

[8] Y. Zhang and J. He, "Correlation between traditional Chinese medicine syndrome elements and relevant factors of essential hypertension," International Journal of Clinical and Experimental Medicine, vol. 12, no. 9, pp. 11851-11858, 2019.

[9] S. Chen, D. Du, Y. Ma, S. Gao, and W. Xiuying, "Clinical study on herbal cone-partitioned moxibustion for irritable bowel syndrome due to spleen-qi deficiency," Journal of Acupuncture and Tuina Science, vol. 9, no. 5, pp. 265-268, 2011.

[10] S. Ling and J. W. Xu, "Model organisms and traditional Chinese medicine syndrome models," Evidence-Based Complementary and Alternative Medicine, vol. 2013, Article ID 761987, 14 pages, 2013.

[11] G. Tian, C. Wu, J. Li et al., "Network pharmacology based investigation into the effect and mechanism of modified Sijunzi decoction against the subtypes of chronic atrophic gastritis," Pharmacological Research, vol. 144, pp. 158-166, 2019.

[12] L. Dai, W. J. Zhou, M. Wang, S. G. Zhou, and G. Ji, “Efficacy and safety of Sijunzi decoction for chronic fatigue syndrome with spleen deficiency pattern: study protocol for a randomized, double-blind, placebo-controlled trial," Annals of Translational Medicine, vol. 7, no. 20, p. 587, 2019.

[13] J. R. Wang, H. Zhou, X. Q. Yi, Z. H. Jiang, and L. Liu, “Total ginsenosides of Radix Ginseng modulates tricarboxylic acid cycle protein expression to enhance cardiac energy metabolism in ischemic rat heart tissues," Molecules, vol. 17, no. 11, pp. 12746-12757, 2012.

[14] E. Hwang, Z. W. Sun, T. H. Lee et al., "Enzyme-processed Korean red ginseng extracts protects against skin damage induced by UVB irradiation in hairless mice," Journal of Ginseng Research, vol. 37, no. 4, pp. 425-434, 2013.

[15] B. Yang, B. Xiao, and T. Sun, "Antitumor and immunomodulatory activity of Astragalus membranaceus polysaccharides in H22 tumor-bearing mice," International Journal of Biological Macromolecules, vol. 62, pp. 287-290, 2013.

[16] A. L. Hopkins, "Network pharmacology: the next paradigm in drug discovery," Nature Chemical Biology, vol. 4, no. 11, pp. 682-690, 2008.

[17] S. Li, "Mapping ancient remedies: applying a network approach to traditional Chinese medicine," Science, vol. 350, no. 6262 , pp. S72-S74, 2015. 
[18] S. Li and B. Zhang, "Traditional Chinese medicine network pharmacology: theory, methodology and application," Chinese Journal of Natural Medicines, vol. 11, no. 2, pp. 110-120, 2013.

[19] J. Zuo, X. Wang, Y. Liu et al., "Integrating network pharmacology and metabolomics study on anti-rheumatic mechanisms and antagonistic effects against methotrexate-induced toxicity of Qing-Luo-Yin," Frontiers in Pharmacology, vol. 9, article 1472, 2018.

[20] H. Li, L. Zhao, B. Zhang et al., "A network pharmacology approach to determine active compounds and action mechanisms of ge-gen-qin-lian decoction for treatment of type 2 diabetes," Evidence-based Complementary and Alternative Medicine, vol. 2014, Article ID 495840, 12 pages, 2014.

[21] X. Wu, R. Jiang, M. Q. Zhang, and S. Li, "Network-based global inference of human disease genes," Molecular Systems Biology, vol. 4, no. 1, p. 189, 2008.

[22] A. L. Barabasi, N. Gulbahce, and J. Loscalzo, "Network medicine: a network-based approach to human disease," Nature Reviews Genetics, vol. 12, no. 1, pp. 56-68, 2011.

[23] J. C. Guo, P. Zhang, L. Zhou et al., "Prognostic and predictive value of a five-molecule panel in resected pancreatic ductal adenocarcinoma: a multicentre study," eBioMedicine, vol. 55, article 102767, 2020.

[24] The Gene Ontology Consortium, "The Gene Ontology Resource: 20 years and still GOing strong," Nucleic Acids Research, vol. 47, no. D1, pp. D330-D338, 2019.

[25] M. Kanehisa and S. Goto, "KEGG: Kyoto Encyclopedia of Genes and Genomes," Nucleic Acids Research, vol. 28, no. 1, pp. 27-30, 2000.

[26] C. Li and W. H. Wong, "Model-based analysis of oligonucleotide arrays: expression index computation and outlier detection," Proceedings of the National Academy of Sciences of the United States of America, vol. 98, no. 1, pp. 31-36, 2001.

[27] R. Edgar, M. Domrachev, and A. E. Lash, "Gene Expression Omnibus: NCBI gene expression and hybridization array data repository," Nucleic Acids Research, vol. 30, no. 1, pp. 207-210, 2002.

[28] J. J. Zhou, G. R. Xie, and X. J. Yan, Chemical Composition of Traditional Chinese Medicine, Science Press, 2009.

[29] C. R. Zhang, G. Cao, X. Cong, Y. Zhang, and B. C. Cai, "Advances in studies on chemistry and quality control of Atractylodes macrocephala koidz," China Journal of Traditional Chinese Medicine and Pharmacy, vol. 26, no. 10, pp. 146-149, 2011.

[30] G. H. Chen and W. F. Huang, "Progress in pharmacological effects of compositions of Astragalus membranaceus," Chinese Journal of New Drugs, vol. 17, no. 17, pp. 1482-1485, 2008.

[31] H. Zhao, X. L. Xie, and J. Xu, "Advancement in studies of chemical constituents and pharmacological activities of Chinese yam," Pharmacy Today, vol. 19, no. 3, pp. 49-52, 2009.

[32] E. Y. Zhu, Q. He, Z. T. Wang, L. S. Xu, and G. J. Xu, "Chemical study on the root of Codonopsis pilosula," Journal of China Pharmaceutical University, vol. 32, no. 2, pp. 94-95, 2001.

[33] G. R. Bickerton, G. V. Paolini, J. Besnard, S. Muresan, and A. L. Hopkins, "Quantifying the chemical beauty of drugs," Nature Chemistry, vol. 4, no. 2, pp. 90-98, 2012.

[34] S. W. Zhao and S. Li, "Network-based relating pharmacological and genomic spaces for drug target identification," PLOS One, vol. 5, no. 7, article e11764, 2010.

[35] X. J. Liang, H. Y. Li, and S. Li, "A novel network pharmacology approach to analyse traditional herbal formulae: the Liu-Wei-
Di-Huang pill as a case study," Molecular BioSystems, vol. 10, no. 5, pp. 1014-1022, 2014.

[36] L. R. Fu, S. W. Guo, and X. H. Liu, "Effect of Yiqi Jianpi plus anticancer herbs on spleen deficiency in colorectal cancer and its anti-tumor role," Asian Pacific Journal of Tropical Medicine, vol. 7, no. 5, pp. 378-381, 2014.

[37] J. Wang, X. J. Xiong, and X. C. Yang, "Expert consensus on diagnosis and treatment of hypertension with traditional Chinese medicine," Chinese Journal of Experimental Traditional Medical Formulae, vol. 25, no. 15, pp. 217-221, 2019.

[38] M. J. Hossen, J. Y. Chou, S. M. Li et al., "An ethanol extract of the rhizome of Atractylodes chinensis exerts anti-gastritis activities and inhibits Akt/NF- $\kappa \mathrm{B}$ signaling," Journal of Ethnopharmacology, vol. 228, pp. 18-25, 2019.

[39] L. L. Tao, Y. Lei, G. L. Wang, L. Q. Zhu, and Y. Wang, "Effect of extracts from Radix Ginseng, Radix Notoginseng and Rhizoma Chuanxiong on delaying aging of vascular smooth muscle cells in aged rats," Chinese Journal of Integrative Medicine, vol. 18, no. 8, pp. 582-590, 2012.

[40] Y. Liu, W. Weng, R. Gao, and Y. Liu, "New insights for cellular and molecular mechanisms of aging and aging-related diseases: herbal medicine as potential therapeutic approach," Oxidative Medicine and Cellular Longevity, vol. 2019, Article ID 4598167, 25 pages, 2019.

[41] S. Zhao and R. Iyengar, "Systems pharmacology: network analysis to identify multiscale mechanisms of drug action," Annual Review of Pharmacology and Toxicology, vol. 52, no. 1, pp. 505-521, 2012.

[42] S. Li, B. Zhang, and N. Zhang, "Network target for screening synergistic drug combinations with application to traditional Chinese medicine," BMC Systems Biology, vol. 5, Supplement 1, p. S10, 2011.

[43] G. C. Huang, L. S. Wu, L. G. Chen, L. L. Yang, and C. C. Wang, "Immuno-enhancement effects of Huang Qi Liu Yi Tang in a murine model of cyclophosphamide-induced leucopenia," Journal of Ethnopharmacology, vol. 109, no. 2, pp. 229-235, 2007.

[44] H. D. Xu, C. G. You, R. L. Zhang, P. Gao, and Z. R. Wang, "Effects of Astragalus polysaccharides and astragalosides on the phagocytosis of Mycobacterium tuberculosis by macrophages," The Journal of International Medical Research, vol. 35, no. 1, pp. 84-90, 2016.

[45] Y. P. Wang, X. Y. Li, C. Q. Song, and Z. B. Hu, "Effect of astragaloside IV on T, B lymphocyte proliferation and peritoneal macrophage function in mice," Acta Pharmacologica Sinica, vol. 23, no. 3, pp. 263-266, 2002.

[46] N. Zhao, W. Zhang, Y. Guo et al., "Effects on neuroendocrinoimmune network of Lizhong pill in the reserpine induced rats with spleen deficiency in traditional Chinese medicine," Journal of Ethnopharmacology, vol. 133, no. 2, pp. 454-459, 2011.

[47] A. Caillon, P. Paradis, and E. L. Schiffrin, "Role of immune cells in hypertension," British Journal of Pharmacology, vol. 176, no. 12, pp. 1818-1828, 2019.

[48] M. N. Peraldi, J. Berrou, N. Dulphy et al., "Oxidative stress mediates a reduced expression of the activating receptor NKG2D in NK cells from end-stage renal disease patients," Journal of Immunology, vol. 182, no. 3, pp. 1696-1705, 2009.

[49] A. Guillaumet-Adkins, Y. Yanez, M. D. Peris-Diaz, I. Calabria, C. Palanca-Ballester, and J. Sandoval, "Epigenetics and oxidative stress in aging," Oxidative Medicine and Cellular Longevity, vol. 2017, Article ID 9175806, 8 pages, 2017. 
[50] J. Nie and S. Sun, "Research on spleen-qi deficiency syndrome of traditional Chinese medicine in recent 10 years," Acta Chinese Medicine and Phamacology, vol. 39, no. 1, pp. 128-130, 2011.

[51] H. You, Y. Lu, D. Gui, A. Peng, J. Chen, and Y. Gu, “Aqueous extract of Astragali Radix ameliorates proteinuria in adriamycin nephropathy rats through inhibition of oxidative stress and endothelial nitric oxide synthase," Journal of Ethnopharmacology, vol. 134, no. 1, pp. 176-182, 2011.

[52] A. G. W. Gong, R. Duan, H. Y. Wang et al., "Evaluation of the pharmaceutical properties and value of astragali radix," Medicine, vol. 5, no. 2, p. 46, 2018.

[53] X. Xiong, G. Huang, and H. Huang, "The antioxidant activities of phosphorylated polysaccharide from native ginseng," International Journal of Biological Macromolecules, vol. 126, pp. 842-845, 2019.

[54] M. Wang, S. J. Yan, H. T. Zhang et al., "Ginsenoside Rh2 enhances the antitumor immunological response of a melanoma mice model," Oncology Letters, vol. 13, no. 2, pp. 681$685,2017$.

[55] M. T. Greenwood, "Dysbiosis, spleen qi, phlegm, and complex difficulties," Medical Acupuncture, vol. 29, no. 3, pp. 128-137, 2017. 